\title{
An ALMA Survey of CO Isotopologue Emission from Protoplanetary Disks in Chamaeleon I
}

\author{
Feng Long (龙凤) $)^{1,2}$, Gregory J. Herczeg (沈雷歌) ${ }^{1}$, Ilaria Pascucci ${ }^{3,4}$, Emily Drabek-Maunder ${ }^{5}$, Subhanjoy Mohanty ${ }^{5}$, \\ Leonardo Testi ${ }^{6}$, Daniel Apai ${ }^{3,4,7}$, Nathan Hendler ${ }^{3}$, Thomas Henning ${ }^{8}$, Carlo F. Manara ${ }^{9}$, and Gijs D. Mulders ${ }^{3,4}$ \\ ${ }^{1}$ Kavli Institute for Astronomy and Astrophysics, Peking University, Yi He Yuan Lu 5, Haidian Qu, 100871 Beijing, China; longfeng@pku.edu.cn \\ ${ }^{2}$ Department of Astronomy, School of Physics, Peking University, 100871 Beijing, China \\ ${ }^{3}$ Lunar and Planetary Laboratory, The University of Arizona, Tucson, AZ 85721, USA \\ ${ }^{4}$ Earths in Other Solar Systems Team, NASA Nexus for Exoplanet System Science \\ ${ }^{5}$ Imperial College London, London SW7 2AZ, UK \\ ${ }^{6} \mathrm{ESO} /$ European Southern Observatory, Garching bei München, Germany \\ ${ }^{7}$ Steward Observatory, The University of Arizona, Tucson, AZ 85721, USA \\ ${ }^{8}$ Max Planck Institute for Astronomy, Heidelberg, Germany \\ ${ }^{9}$ Scientific Support Office, Directorate of Science, European Space Research and Technology Centre (ESA/ESTEC), Noordwijk, The Netherlands \\ Received 2017 January 20; revised 2017 May 28; accepted 2017 June 8; published 2017 July 26
}

\begin{abstract}
The mass of a protoplanetary disk limits the formation and future growth of any planet. Masses of protoplanetary disks are usually calculated from measurements of the dust continuum emission by assuming an interstellar gas-todust ratio. To investigate the utility of $\mathrm{CO}$ as an alternate probe of disk mass, we use ALMA to survey ${ }^{13} \mathrm{CO}$ and $\mathrm{C}^{18} \mathrm{O} J=3-2$ line emission from a sample of 93 protoplanetary disks around stars and brown dwarfs with masses from 0.03 to $2 M_{\odot}$ in the nearby Chamaeleon I star-forming region. We detect ${ }^{13} \mathrm{CO}$ emission from 17 sources and $\mathrm{C}^{18} \mathrm{O}$ from only one source. Gas masses for disks are then estimated by comparing the $\mathrm{CO}$ line luminosities to results from published disk models that include $\mathrm{CO}$ freeze-out and isotope-selective photodissociation. Under the assumption of a typical interstellar medium CO-to- $\mathrm{H}_{2}$ ratio of $10^{-4}$, the resulting gas masses are implausibly low, with an average gas mass of $\sim 0.05 M_{\text {Jup }}$ as inferred from the average flux of stacked ${ }^{13} \mathrm{CO}$ lines. The low gas masses and gas-to-dust ratios for Cha I disks are both consistent with similar results from disks in the Lupus starforming region. The faint $\mathrm{CO}$ line emission may instead be explained if disks have much higher gas masses, but freeze-out of $\mathrm{CO}$ or complex $\mathrm{C}$-bearing molecules is underestimated in disk models. The conversion of $\mathrm{CO}$ flux to $\mathrm{CO}$ gas mass also suffers from uncertainties in disk structures, which could affect gas temperatures. $\mathrm{CO}$ emission lines will only be a good tracer of the disk mass when models for $\mathrm{C}$ and $\mathrm{CO}$ depletion are confirmed to be accurate.
\end{abstract}

Key words: protoplanetary disks - stars: pre-main sequence - submillimeter: planetary systems

\section{Introduction}

The formation and migration of planets within protoplanetary disks likely contribute to the rich diversity of exoplanet architectures (see review by Winn \& Fabrycky 2015). The initial disk mass, the evolution of the gas-to-dust ratio, the evolution of gas surface density distribution with time, and the gas dispersal timescale all influence how many and what types of planets can form in a stellar system (e.g., Pollack et al. 1996; Boss 1997; Mordasini et al. 2012; Alexander et al. 2014). Statistical studies reveal possible correlations between stellar mass and planet occurrence rates, including a higher frequency of small planets in close orbits around M dwarfs than around FGK stars (Howard et al. 2012; Mulders et al. 2015a) and a higher occurrence rate of giant planets around solar-type stars than around $\mathrm{M}$ dwarfs (Johnson et al. 2010), although this correlation may not apply to hot Jupiters (Obermeier et al. 2016). While these relationships likely originate in correlations between stellar mass and disk properties, linking disk properties to the outcome of planet formation requires an unbiased census of initial disk masses and the evolution of disk mass and structure with time.

Recent submillimeter surveys of disks demonstrate that the dust mass in disks is strongly correlated with stellar mass in individual regions with ages between 1 and $10 \mathrm{Myr}$ (Andrews et al. 2013; Mohanty et al. 2013; Ansdell et al. 2016, 2017; Barenfeld et al. 2016; Pascucci et al. 2016). The correlation of dust mass with stellar mass is steeper in the 5-10 Myr Upper
Sco association than in the younger regions, which when combined with a much lower fraction of stars with disks in Upper Sco (Luhman \& Mamajek 2012) suggests that dust in disks evolve quickly and with a dependence on the mass of the central star. However, the estimates of dust mass rely on the dust opacity, which depends on the dust grain size distribution and composition (e.g., Beckwith et al. 2000) and on the assumed dust temperature (e.g., Andrews et al. 2013; Pascucci et al. 2016; van der Plas et al. 2016). In addition, the total disk mass is usually converted from dust mass using a gas-to-dust ratio of $\sim 100$ for the interstellar medium (ISM; Bohlin et al. 1978). During disk evolution, gas and dust may decouple from each other, as seen in differences in their spatial distributions in some disks (e.g., Isella et al. 2007; Andrews et al. 2012; van der Marel et al. 2013; Perez et al. 2015).

Independent gas mass measurements are needed as observational evidence to better understand gas and dust evolution. Because $\mathrm{H}_{2}$ does not emit at cold temperatures, $\mathrm{CO}$ isotopologues are widely used to probe the gas content of disks. CO chemistry has been studied extensively, and the pure rotational CO lines are readily detectable at millimeter wavelengths (Henning \& Semenov 2013). However, as with dust, the conversion of a $\mathrm{CO}$ line luminosity to a total gas mass is plagued by uncertainties, primarily in the gas-phase CO-to- $\mathrm{H}_{2}$ abundance ratio and in isotopologue ratios. Even for $\mathrm{C}^{18} \mathrm{O}$, line opacities can be high in some disks (e.g., Yu et al. 2017). 
CO gas depletes through freeze-out at the disk midplane (e.g., Dutrey et al. 1997; van Zadelhoff et al. 2001), reducing the CO-to- $\mathrm{H}_{2}$ abundance ratio. $\mathrm{CO}$ gas is also photodissociated in the warm surface layer of the disk (e.g., Aikawa et al. 2002; Kamp \& Dullemond 2004; Gorti et al. 2011; Walsh et al. 2012), in which the less abundant $\mathrm{C}^{18} \mathrm{O}$ and ${ }^{13} \mathrm{CO}$ are selectively dissociated by ultraviolet radiation, while ${ }^{12} \mathrm{CO}$ may effectively self-shield. This isotope-selective photodissociation therefore modifies the abundance ratios of the $\mathrm{CO}$ isotopologues and the conversion from rare isotopologues to the CO gas mass (van Dishoeck \& Black 1988; Lyons \& Young 2005; Smith et al. 2009).

When $\mathrm{CO}$ freeze-out and isotope-selective photodissociation are implemented into chemical models of disks, the conversion between line luminosities and disk mass changes by as much as two orders of magnitude (e.g., Woitke et al. 2009; Miotello et al. 2014). Miotello et al. (2016, hereafter MvD16) provides conversion factors from $\mathrm{CO}$ isotopologue line luminosity to disk mass, calculated from thermochemical models of static disks that include freeze-out and isotope-selective photodissociation, as implemented from the DALI code (Bruderer 2013; Miotello et al. 2014). In an earlier, simplified approach, Williams \& Best (2014, hereafter WB14) developed parametric models of disk properties, constraining the $\mathrm{CO}$ freeze-out at $T<20 \mathrm{~K}$ and estimating isotope-selective photodissociation by a reduction of $\mathrm{C}^{18} \mathrm{O}$ abundance. The gas mass is then derived by comparing the observed $\mathrm{CO}$ isotopologue line luminosity with their simulated line luminosity.

A possible missing ingredient in these static disk models is carbon depletion into complex molecules, which may then freeze out. Carbon depletion, in excess of that estimated from freeze-out or photosdissociation, is inferred from the comparison of HD and CO gas mass (Bergin et al. 2013; Favre et al. 2013; McClure et al. 2016; Schwarz et al. 2016) and from observations of neutral C and $\mathrm{CO}$ emission (Kama et al. 2016a). To account for these discrepancies, elemental carbon may be sequestered from $\mathrm{CO}$ into more complex carbon chains or $\mathrm{CO}_{2}$, which is then locked up in ice grains (Favre et al. 2013; Du et al. 2015; Kama et al. 2016b). Similar depletion processes also apply to oxygen volatiles, as suggested from water observations (e.g., Du et al. 2017). The depletion of $\mathrm{CO}$ and other volatiles may be accelerated by vertical mixing, because the turbulence in the midplane is much lower than at the disk surface (Krijt et al. 2016; Xu et al. 2017).

In this work, we use ALMA to survey ${ }^{13} \mathrm{CO}$ and $\mathrm{C}^{18} \mathrm{O}$ line emission from disks in the young ( 2 Myr; Luhman 2004), nearby $(188 \pm 12 \mathrm{pc} \text {; see Appendix A })^{10}$ Chamaeleon I starforming region, hereafter Cha I. Our results and interpretations are broadly consistent with the analysis of $\mathrm{CO}$ emission from disks in the Lupus star-forming region by Ansdell et al. (2016) and recent interpretation of those data by Miotello et al. (2017). We first describe our ALMA observations and data reduction in Section 2. The methods developed for measuring the line fluxes and upper limits are presented in Section 3. The CO gas properties and disk mass inferred from the weak $\mathrm{CO}$ emission and disk models are described in Sections 4-5. We compare our results with other starforming regions and discuss disk evolution and its implications for planet formation theories in Sections 6.1 and 6.2. We summarize our findings in Section 7.

\footnotetext{
10 The companion paper Pascucci et al. (2016) used a pre-Gaia distance of $160 \mathrm{pc}$. In this paper, all luminosities and disk masses are recalculated using this updated distance. The stellar masses adopted from Pascucci et al. (2016) are not recalculated in this paper, since most of our sample are low-mass stars on the vertical Hayashi tracks.
}

\section{ALMA Observations}

We use ALMA Band 7 to survey the Class II protoplanetary disks in Cha I in the Cycle 2 program 2013.1.00437 (PI: I. Pascucci). The sample was split into shallow observations of 54 stars with spectral type (SpTy) equal to or earlier than M3, hereafter referred to as the Hot sample, and deeper observations of 39 stars with later SpTy, referred to as the Cool sample. The sample, observation calibrator setups, and results of the dust continuum are described in detail by Pascucci et al. (2016). One object, 2MASS J11183572-7935548, is likely a member of $\epsilon$ Cha (Luhman et al. 2008; Lopez Martí et al. 2013; Murphy et al. 2013) and has been excluded from the global analyses of disks in this work.

In this paper, we focus on observations from one baseband, which was split into segments centered at $330.6 \mathrm{GHz}$ to target the ${ }^{13} \mathrm{CO} J=3-2$ and at $329.3 \mathrm{GHz}$ to target the $\mathrm{C}^{18} \mathrm{O} J=3-2$. The correlator was configured to cover both lines, each with a bandwidth of $117.2 \mathrm{MHz}$ and a channel separation of $0.122 \mathrm{MHz}$ $\left(0.11 \mathrm{~km} \mathrm{~s}^{-1}\right)$. The three other spectral windows were configured for continuum observations. For each target, two sets of observations were executed for a total integration time of $24 \mathrm{~s}$ per source for the Hot sample and $120 \mathrm{~s}$ per source for the Cool sample. The observations were performed in good weather conditions with a precipitable water vapor of $\sim 0.6-0.9 \mathrm{~mm}$.

The ALMA data are calibrated using the Common Astronomy Software Application (CASA) package and following the data reduction scripts provided by NRAO, including flux, phase, bandpass, and gain calibrations. The absolute flux scale has a systematic uncertainty of $10 \%$. The two executions of each object are then concatenated using the concat task after flux calibration. Continuum emission is subtracted in the $u v$-plane from the spectral windows containing $\mathrm{CO}$ isotopologue lines using the uvcontsub task. The spectral line data cubes are then created with the clean algorithm using the continuum-subtracted visibilities. Considering the weakness of line emission and the low detection rate in our sample, natural weighting is chosen in clean to favor shorter baselines and hence achieve a higher signal-to-noise ratio $(\mathrm{S} / \mathrm{N})$.

In general, longer baseline visibilities trace smaller-scale structures, and shorter baseline visibilities trace extended emission from larger scale. Since our primary objective is to measure the total flux from the disk, all baselines are included in clean. This choice also provides consistency with the dust analysis of the same sample by Pascucci et al. (2016). Excluding the visibilities with baselines $<40 \mathrm{k} \lambda$ would avoid large-scale ripples from any extended emission but will also lower the calculated line fluxes in most cases. In alternate reductions that exclude baselines shorter than $<40 \mathrm{k} \lambda$, the flux differences are within uncertainty in most cases, although the two sources 2MASS J11075792-7738449 and 2MASS J11095340-7634255 would be much fainter. The ${ }^{13} \mathrm{CO}$ detection rates would also not be affected.

The resulting synthesized beam for the $\mathrm{CO}$ data cube is 0 " $7 \times 0$." 5 for both samples, the same as the continuum data. The CO transitions are imaged at a spectral resolution of $0.25 \mathrm{~km} \mathrm{~s}^{-1}$ and reach the $1 \sigma$ noise rms of $30(43) \mathrm{mJy} \mathrm{beam}^{-1}$ and 107 (126) mJy beam ${ }^{-1}$ for ${ }^{13} \mathrm{CO}\left(\mathrm{C}^{18} \mathrm{O}\right)$ in the Cool and Hot samples, respectively. The slightly higher noise in $\mathrm{C}^{18} \mathrm{O}$ may be caused by placing the $\mathrm{C}^{18} \mathrm{O}$ line closer to the band edge than ${ }^{13} \mathrm{CO}$, resulting in a lower instrument response. 

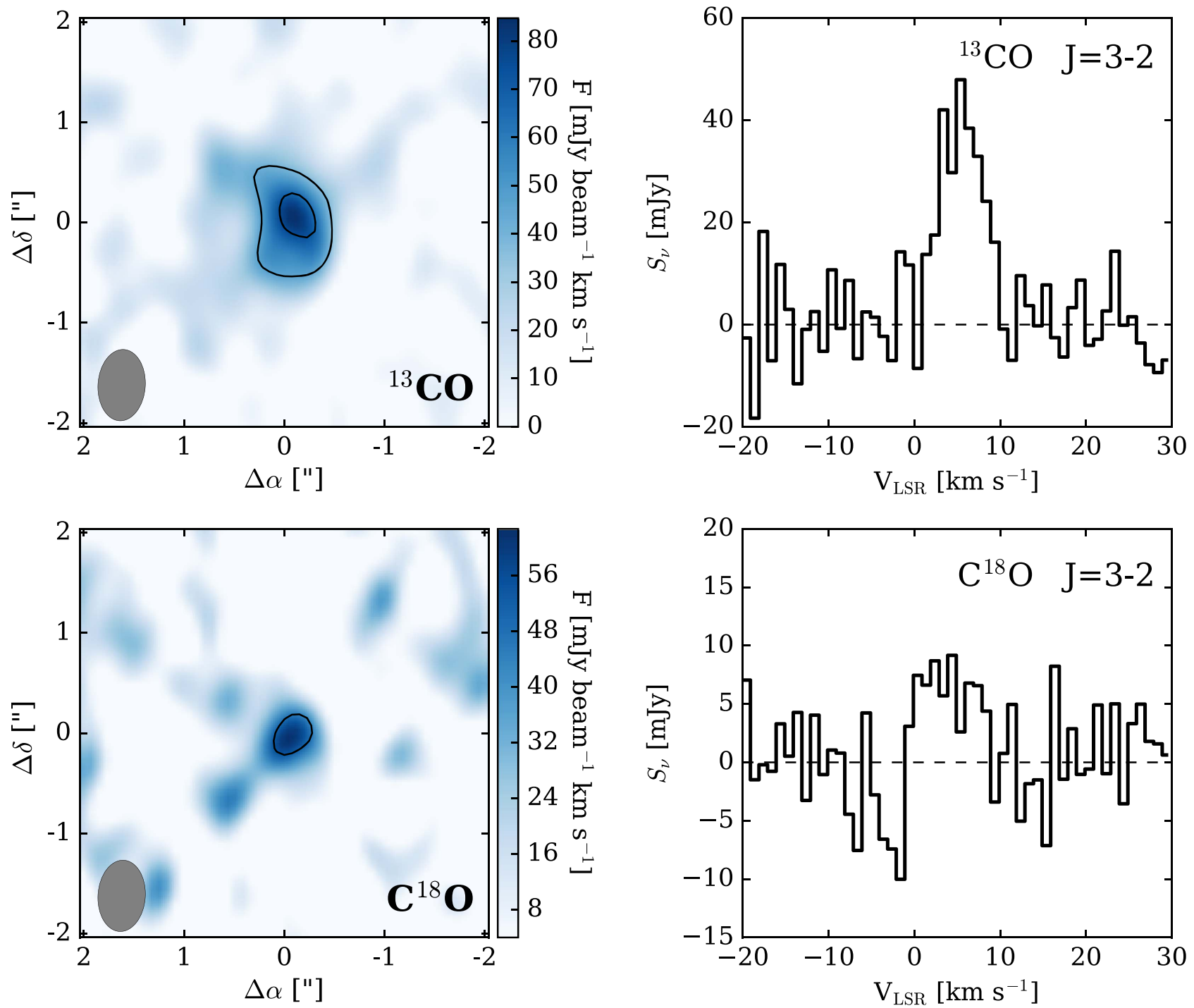

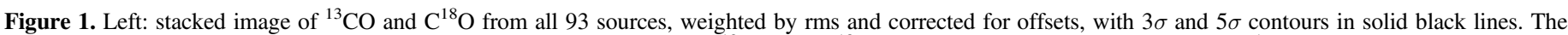
typical beam size is shown in the left corner. Right: stacked spectrum for ${ }^{13} \mathrm{CO}$ and $\mathrm{C}^{18} \mathrm{O}$ from all 93 sources, binned to $1 \mathrm{~km} \mathrm{~s}{ }^{-1}$.

\section{Measuring CO Line Fluxes and Upper Limits}

Only a few strong ${ }^{13} \mathrm{CO}$ detections are easily identified from line profiles. Therefore, we develop a method to uniformly identify weak detections and measure line fluxes, uncertainties, and upper limits from the continuum-subtracted cleaned images. We first stack the images and spectra from the full sample to identify the emission region and velocity range used for uniform flux measurements for each source. The flux uncertainties are calculated by using the full spectral images outside the $\mathrm{CO}$ emission range, which yields lower uncertainties and better consistency within the Hot and Cool samples than if only the $\mathrm{CO}$ spectral channels would have been used. For sources with $\mathrm{CO}$ detections, the extraction regions are adjusted to calculate final fluxes that include the full spatial extent of the emission. The following subsections describe each step in this method.

\subsection{Defining the Extraction Region}

We obtain initial CO images by integrating over the velocity range of $0-10 \mathrm{~km} \mathrm{~s}^{-1}$ over which emission is detected (see
Figure 1, right panels). The images are shifted to the centroid position of the continuum emission by applying the phasecenter offsets from Pascucci et al. (2016), weighted by the noise (not noise squared, so that each observation contributes equally to the final stacked image), and then stacked into a single image. For sources that were not detected in continuum emission, a phase-center (position) offset of -0.13 in right ascension and $0 . \prime 0$ in declination were adopted from the median phase-center offset of continuum detections from Pascucci et al. (2016).

The stacked images for ${ }^{13} \mathrm{CO}$ and $\mathrm{C}^{18} \mathrm{O}$ are shown in the left panels of Figure 1, with the peak S/N per beam of 5.8 and 3.5, respectively. Based on the $3 \sigma$ contours, we adopt extraction radii of $\sim 0$." 6 for ${ }^{13} \mathrm{CO}$ and $0 . " 3$ for $\mathrm{C}^{18} \mathrm{O}$, both centered at the continuum position. Stacked images from the continuumdetected sources yield similar spatial extent but higher peak $\mathrm{S} / \mathrm{N}$. Although ${ }^{13} \mathrm{CO}$ and $\mathrm{C}^{18} \mathrm{O}$ should be emitted from the same disk emission area, the marginal detection in $\mathrm{C}^{18} \mathrm{O}$ leads us to choose smaller extraction radii (with a total aperture size comparable to one beam size) to optimize the detection rate of weak signals. 

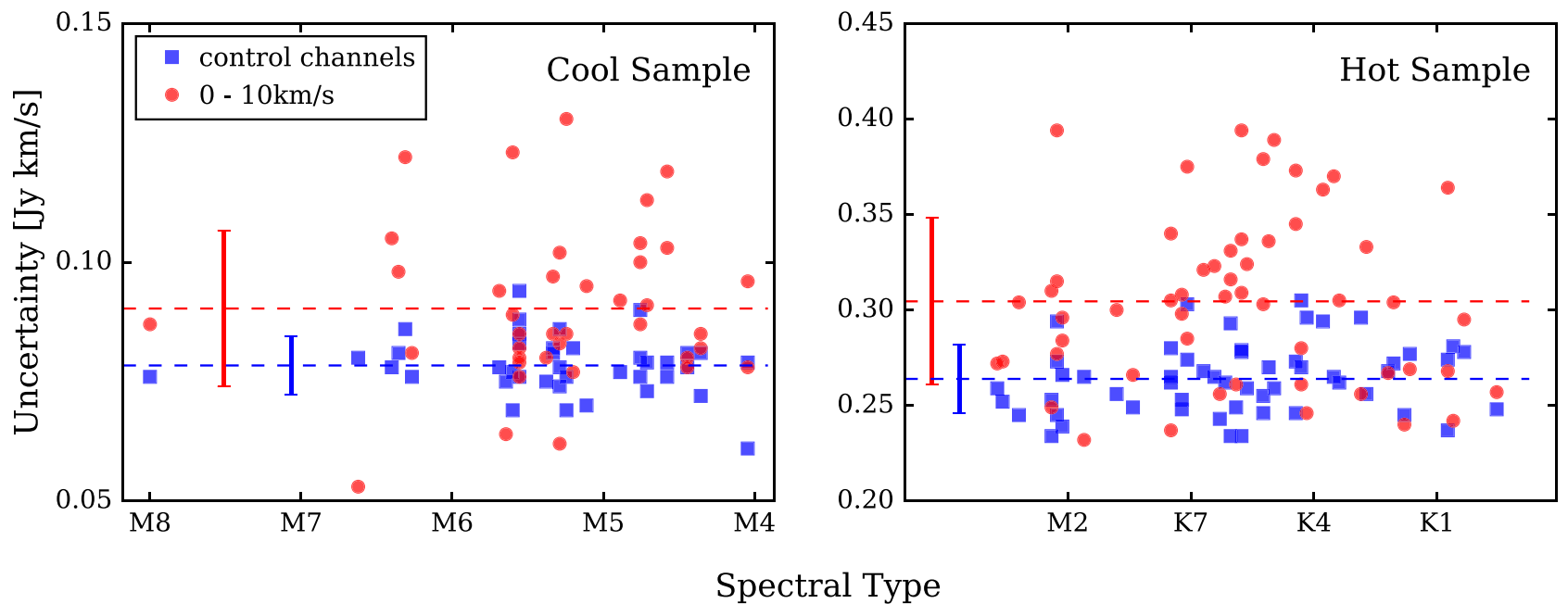

Figure 2. Comparison of uncertainties from 0 to $10 \mathrm{~km} \mathrm{~s}^{-1}$ and mean value of six control channels for the Cool (left) and Hot (right) samples. The mean (dashed line) and $1 \sigma$ standard deviation (solid vertical line) are marked for the $0-10 \mathrm{~km} \mathrm{~s}^{-1}$ range in red and control channels in blue.

Table 1

Uncertainty Comparison

\begin{tabular}{lccccc}
\hline \hline \multirow{2}{*}{ Samples } & \multicolumn{2}{c}{ Mean } & & \multicolumn{2}{c}{ Standard Deviation } \\
\cline { 2 - 3 } \cline { 6 - 6 } & Hot & Cool & & Hot & Cool \\
\hline $0-10 \mathrm{~km} \mathrm{~s}^{-1}$ & 0.304 & 0.090 & & 0.043 & 0.016 \\
Control channels & 0.264 & 0.078 & & 0.018 & 0.006 \\
\hline
\end{tabular}

Note. The mean value and standard deviation of uncertainties in the Hot and Cool samples, in units of $\mathrm{Jy} \mathrm{km} \mathrm{s}^{-1}$.

The spectra are then extracted from these circular apertures centered at the measured or expected continuum source position from Pascucci et al. (2016). Because only a few sources show a clear spectral profile, we also stack all spectra, weighted by rms and binned by four channels to a spectral resolution of $1 \mathrm{~km} \mathrm{~s}^{-1}$. The top right panel of Figure 1 shows that the stacked ${ }^{13} \mathrm{CO}$ emission covers from 0 to $10 \mathrm{~km} \mathrm{~s}^{-1}$, which is adopted as the extraction velocity range for flux calculations. The centroid velocity of ${ }^{13} \mathrm{CO}$ emission of $5.4 \mathrm{~km} \mathrm{~s}^{-1}$ is consistent with the radial velocity measurements of an average LRSK value of $\sim 5 \mathrm{~km} \mathrm{~s}^{-1}$ in Cha I sources (e.g., van Kempen et al. 2009; Nguyen et al. 2012). Since the stacked $\mathrm{C}^{18} \mathrm{O}$ line lacks sufficient $\mathrm{S} / \mathrm{N}$ to measure the spectral profile, we assume that $\mathrm{C}^{18} \mathrm{O}$ and ${ }^{13} \mathrm{CO}$ have the same emission velocity.

\subsection{Measuring the Uncertainties in Fluxes}

The spatial and spectral extraction regions defined in Section 3.1 will be applied in Section 3.3 to measure fluxes and upper limits in the ${ }^{13} \mathrm{CO}$ line in the $0-10 \mathrm{~km} \mathrm{~s}^{-1}$ spectral range and 0 ." 6 radius apertures $\left(0\right.$ " 3 for $\left.\mathrm{C}^{18} \mathrm{O}\right)$. In other studies, the uncertainty is usually measured from the noise level in the same velocity channels as the flux measurements. In this section, we demonstrate that this approach yields large inconsistencies across the sample. We instead develop a method to use the full spectral range to assess uncertainties that are uniform within the Hot and Cool samples.

Uncertainties in fluxes are measured by calculating the standard deviation of fluxes in randomly distributed apertures with the same extraction area and $10 \mathrm{~km} \mathrm{~s}^{-1}$ velocity widths.
The velocity ranges used for uncertainty calculations are spread across the full spectral band in six independent ranges, ${ }^{11}$ hereafter called control channels, and from 0 to $10 \mathrm{~km} \mathrm{~s}^{-1}$, called the emission channels. In each $10 \mathrm{~km} \mathrm{~s}^{-1}$ velocity range from the control channels, fluxes are extracted from 20 circular apertures, each placed at a random central location across the image. In the $0-10 \mathrm{~km} \mathrm{~s}^{-1}$ emission channels, the aperture centers are always located at a distance of two radii from the source center.

The mean value and standard deviation of uncertainties from each sample are shown in Figure 2 and Table 1. When only the $0-10 \mathrm{~km} \mathrm{~s}^{-1}$ range is used, the standard deviation of the uncertainties is 2.5 times higher than if the control channels are used. The large scatter of uncertainties in the $0-10 \mathrm{~km} \mathrm{~s}^{-1}$ channels would render any uniform approach invalid, either by missing detections owing to an anomalously high flux uncertainty or by yielding false detections when the uncertainty is underestimated. The uncertainty difference may be the result of the clean process, during which the emission structure can introduce additional pattern noise spreading in these channels, although in a few cases the noise is lower in the emission channels. Therefore, we adopt the mean value from the six control channels as the flux uncertainty for each source to provide better consistency in the calculated flux uncertainties between objects.

\subsection{Quantifying the Significance of Detections}

To simulate a false detection probability and to determine the confidence level for detections, we compare the flux measurements centered on the $\mathrm{CO}$ lines with background fluxes from the six control channels for each of the 93 targets. The background fluxes are calculated within the measured (or expected) continuum center in each $10 \mathrm{~km} \mathrm{~s}^{-1}$ velocity range from the control channels with a $0 .{ }^{\prime \prime} 6$ radius aperture in the ${ }^{13} \mathrm{CO}$ data cube $\left(0\right.$ ". 3 for $\left.\mathrm{C}^{18} \mathrm{O}\right)$, resulting in a total sample of 558 fluxes. The uncertainty for each target is adopted as discussed above. The normalized histograms of flux-to-uncertainty ratios $(\mathrm{S} / \mathrm{N})$ in $0-10 \mathrm{~km} \mathrm{~s}^{-1}$ and in the control channels are shown in Figure 3 . The 558 data points from the control channels in ${ }^{13} \mathrm{CO}$

\footnotetext{
$\overline{11}$ The six velocity ranges are -45 to $-35,-30$ to $-20,-15$ to $-5,15$ to 25 , 25 to 35 , and 35 to $45 \mathrm{~km} \mathrm{~s}^{-1}$.
} 

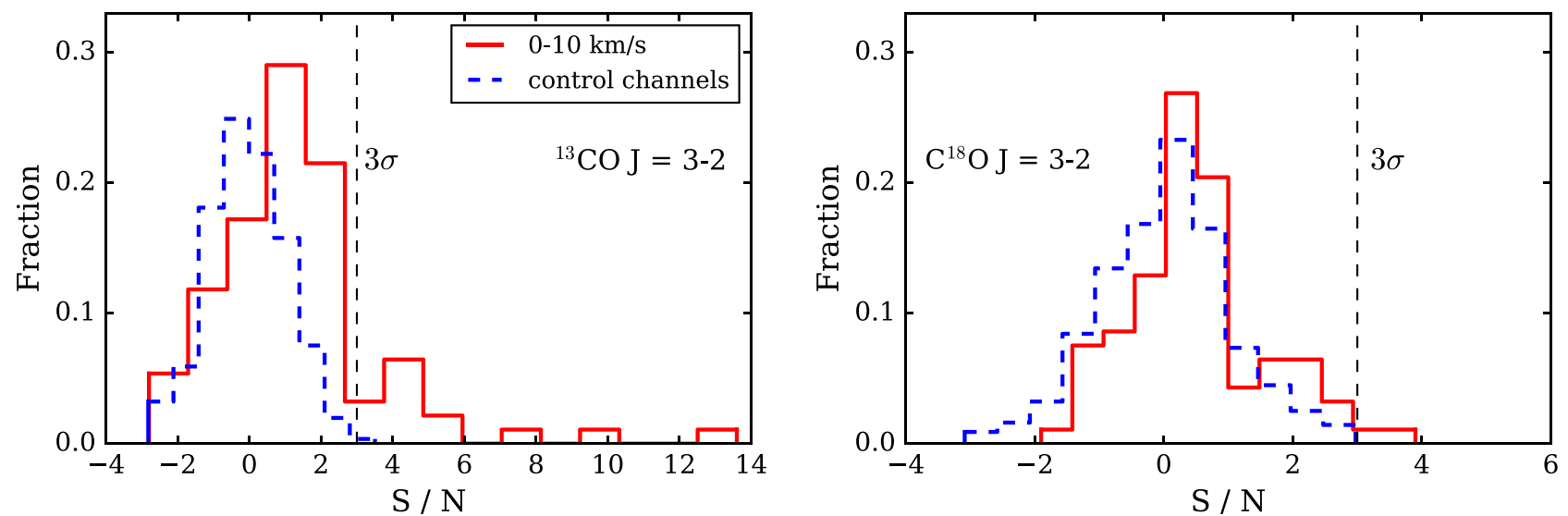

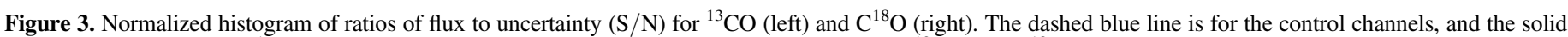
red line is for $0-10 \mathrm{~km} \mathrm{~s}^{-1}$ channels. The vertical dashed lines indicate the $3 \sigma$ significance in ${ }^{13} \mathrm{CO}$ and $\mathrm{C}^{18} \mathrm{O}$.

and in $\mathrm{C}^{18} \mathrm{O}$ are distributed in a Gaussian-like shape. Only one of $558{ }^{13} \mathrm{CO}$ and two of $558 \mathrm{C}^{18} \mathrm{O}$ data points have $\mathrm{S} / \mathrm{N}$ larger than 3 , consistent with the expectation for the frequency of a $3 \sigma$ deviation from the mean in a Gaussian distribution. Therefore, we define ${ }^{13} \mathrm{CO}$ and $\mathrm{C}^{18} \mathrm{O}$ detections as any source exceeding $3 \sigma$ significance from the $0-10 \mathrm{~km} \mathrm{~s}^{-1}$ emission channels, respectively. With this cutoff, detections have a confidence level of $\sim 99.8 \%$.

Fluxes are then measured in the ${ }^{13} \mathrm{CO}$ line using a 0. . 6 radius aperture $\left(0\right.$. . 3 radius aperture for $\left.\mathrm{C}^{18} \mathrm{O}\right)$, and with uncertainties applied from the analysis of the control channels. The distribution of $\mathrm{S} / \mathrm{N}$ in the ${ }^{13} \mathrm{CO}$ line includes a long tail exceeding $3 \sigma$. In the distribution of $\mathrm{C}^{18} \mathrm{O}$ fluxes, only one $4 \sigma$ outlier stands out as a significant detection. Furthermore, while the distributions in the control channels are centered at zero significance, the peak positions of the distributions in the $0-10 \mathrm{~km} \mathrm{~s}^{-1}$ range fall at $1.5 \sigma$ and $0.5 \sigma$ in ${ }^{13} \mathrm{CO}$ and $\mathrm{C}^{18} \mathrm{O}$, respectively. Many sources likely have ${ }^{13} \mathrm{CO}$ fluxes that are just below our detection limits and would have been detected with 2-4 times better sensitivity.

\subsection{CO Line Fluxes and Detections}

Following the methods described in Sections 3.1-3.3, fluxes and uncertainties are measured consistently across our sample over a uniform spatial area and velocity range. Table 2 lists the measured fluxes and uncertainties for both ${ }^{13} \mathrm{CO}$ and $\mathrm{C}^{18} \mathrm{O}$ emission from each source, along with the flux-to-uncertainty ratios for ${ }^{13} \mathrm{CO}$ when a smaller $(0$ ". 3$)$ extraction radius is used to avoid missing weak emission or small disks. In the stacked ${ }^{13} \mathrm{CO}$ image, the flux would be a factor of 2.5 lower if the 0.13 extraction radius were used instead of the 0 " 6 radius. Because the uncertainty increases with larger aperture size, in several cases the flux is significant when extracted from the smaller aperture and below significance when extracted from the larger aperture. The opposite may also occur when the emission is spread over large areas. Therefore, we classify sources as significant ${ }^{13} \mathrm{CO}$ detections if the flux exceeds $3 \sigma$ significance when extracted from both apertures. Sources are considered tentative ${ }^{13} \mathrm{CO}$ detections if the flux exceeds $\sim 3 \sigma$ only in one aperture or is nearly $3 \sigma$ in both apertures. 2MASS J11100010-7634578 has ${ }^{13} \mathrm{CO}$ fluxes that are less than $3 \sigma$ in both apertures for our uniform CO flux measurement but is considered a significant detection because the emission is spatially extended beyond the 0 .' 6 radius aperture. In addition, some sources with weak emission and a narrow line width may be missed in our analysis when the $0-10 \mathrm{~km} \mathrm{~s}^{-1}$ velocity range is uniformly used, since the uncertainty would increase when more channels are included.

For the sources classified as detections, the method adopted above to measure fluxes uniformly across the sample needs to be adjusted to account for different spatial sizes and line profiles of the $\mathrm{CO}$ emission. To determine the size of the ${ }^{13} \mathrm{CO}$ emission region, we generate intensity maps (zero-moment maps) by integrating over the $0-10 \mathrm{~km} \mathrm{~s}^{-1}$ velocity range. We then measure the fluxes by increasing the circular aperture size in 0 ". 15 radius increments on the intensity map until the flux flattens. Since the increasing flux in a larger aperture could be attributed to either real disk signal or noise fluctuations, the real disk size is determined after inspecting the intensity map. For each individual detection, the resulting source size is used as the extraction aperture for flux measurements and is shown as a dashed circle on the intensity map in Figure 10. In the extracted spectra, most ${ }^{13} \mathrm{CO}$ detections display weak emission across the $0-10 \mathrm{~km} \mathrm{~s}^{-1}$ velocity range as shown in Figure 10. Two exceptions, 2MASS J11004022-7619280 and 2MASS J11075792-7738449, exhibit narrow-line emission with FWHM $\sim 1.5 \mathrm{~km} \mathrm{~s}^{-1}$. For each detection, the final flux and uncertainty measurements are extracted from these updated spatial and spectral regions of the ${ }^{13} \mathrm{CO}$ emission, as listed in Table 3.

For the ${ }^{13} \mathrm{CO}$ nondetections, which are below $3 \sigma$ significance in both apertures, the $3 \sigma$ contour size in the stacked image is smaller than the beam size. It is therefore reasonable to choose the $0 . \prime 3$ radius aperture (comparable to one beam size) for nondetections to lower the ${ }^{13} \mathrm{CO}$ upper limits. However, such a small aperture may miss emission from large disks with low surface density. While we adopt upper limits for ${ }^{13} \mathrm{CO}$ nondetections from 0 ." 6 radius aperture results, we also discuss the lower upper limits obtained from smaller extraction regions when results depend on fluxes.

We also excluded the shortest baselines and repeated this full analysis. Most fluxes and uncertainties are similar, with two exceptions: the extended nebulosity near 2MASS J11075792-7738449 (see Appendix B.1.2) and the large disk around 2MASS J11095340-7634255.

\section{Analysis of CO Gas Detections}

In this section, we provide an overview of the general properties of the ${ }^{13} \mathrm{CO}$ and $\mathrm{C}^{18} \mathrm{O}$ emission, including fluxes and 

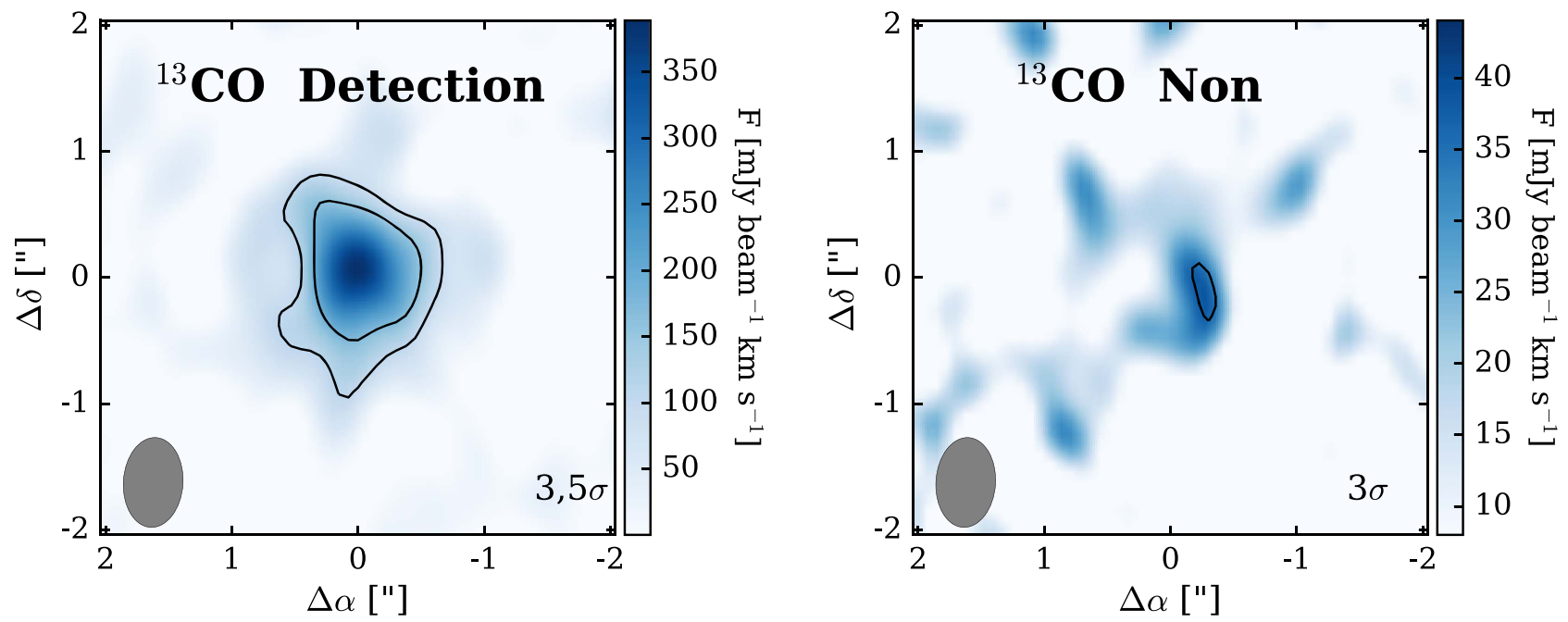

Figure 4. Stacked ${ }^{13} \mathrm{CO}$ image for ${ }^{13} \mathrm{CO}$ detections (left) and nondetections (right), with $3 \sigma$ and $5 \sigma$ contours in solid black lines. The typical beam size is shown in the left corner.
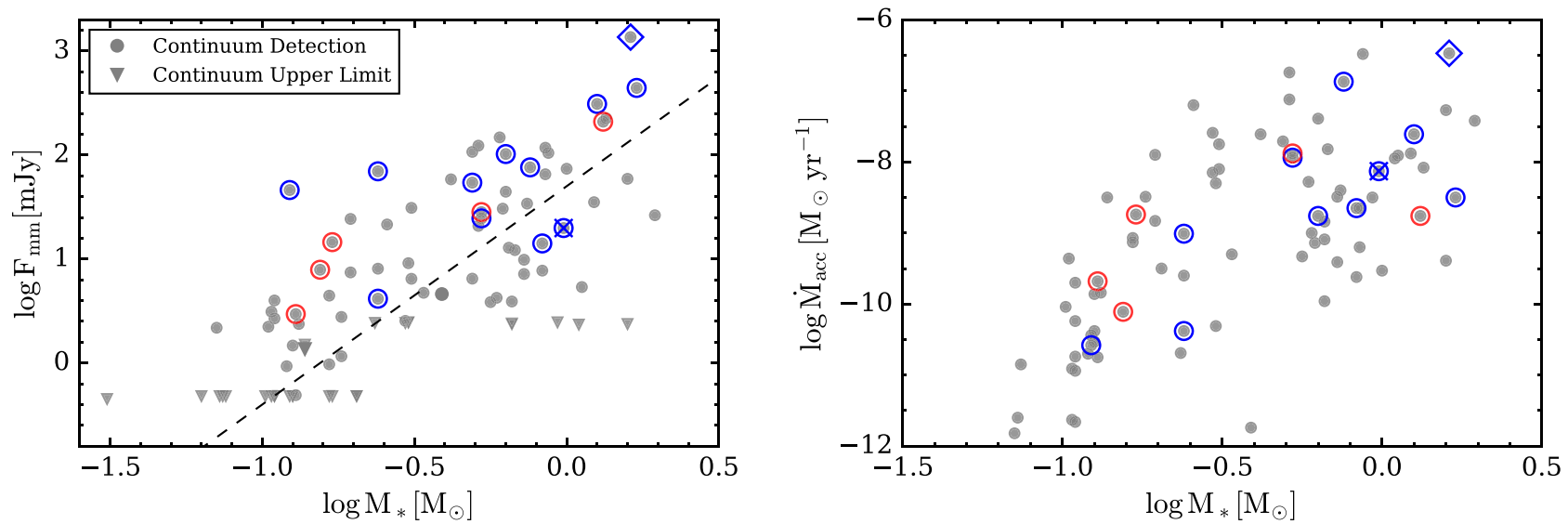

Figure 5. Left: $887 \mu \mathrm{m}$ continuum flux densities $\left(F_{\mathrm{mm}}\right)$ vs. stellar mass $\left(M_{*}\right)$; data and best-fit relation plotted as a dashed $\operatorname{line}\left[\log \left(F_{\mathrm{mm}} / \mathrm{mJy}\right)=1.9( \pm 0.2) \times \log \right.$ $\left.\left(M_{*} / M_{\odot}\right)+1.6( \pm 0.1)\right]$ are adopted from Pascucci et al. (2016); see also Table 2. Gray circles are continuum detections, while downward-pointing triangles stand for upper limits for nondetections. Significant (12) and tentative $(5){ }^{13} \mathrm{CO}$ detections are marked in blue and red, respectively. The strongest continuum source, $2 \mathrm{MASS}$ $\mathrm{J} 11100010-7634578$, detected in both ${ }^{13} \mathrm{CO}$ and $\mathrm{C}^{18} \mathrm{O}$, is noted with a diamond. 2MASS J11075792-7738449, with off-source ${ }^{13} \mathrm{CO}$ emission, is marked with a cross. Right: Accretion rate for 82 sources (Manara et al. 2016a, 2017) vs. stellar mass (Pascucci et al. 2016).

spatial distributions. We then investigate possible correlations with dust mass in the disk, stellar mass, and accretion rate.

\subsection{The General Properties of CO Detections}

Out of 93 disks in our sample, 12 are classified as significant detections and 5 as tentative detections in ${ }^{13} \mathrm{CO} J=3-2$. The source 2MASS J11075792-7738449 shows extended ${ }^{13} \mathrm{CO}$ emission and extremely high ${ }^{13} \mathrm{CO}$ flux, which is likely associated with the reflection nebulosity and is excluded from the full sample analysis (see also Appendix B.1.2). Only the strongest continuum source in our sample, 2MASS J111000107634578 , is detected in both ${ }^{13} \mathrm{CO}$ and $\mathrm{C}^{18} \mathrm{O}$. Another source, 2MASS J11065939-7530559, is tentatively detected in $\mathrm{C}^{18} \mathrm{O}$ at $3 \sigma$ but is rejected owing to the absence of ${ }^{13} \mathrm{CO}$ emission. All of the significant and tentative ${ }^{13} \mathrm{CO}$ detections are also detected in continuum emission at $887 \mu \mathrm{m}$ (Pascucci et al. 2016).

For the only detection in $\mathrm{C}^{18} \mathrm{O}, 2 \mathrm{MASS} \mathrm{J} 11100010$ 7634578, the flux ratio of ${ }^{13} \mathrm{CO}$ to $\mathrm{C}^{18} \mathrm{O}$ line emission is $\sim 1-2$, much lower than expected from their isotopologue ratio of $\sim 8$ (Wilson \& Rood 1994). This indicates that ${ }^{13} \mathrm{CO}$ is likely optically thick (see discussion in Appendix B.1.1). No other source is detected in $\mathrm{C}^{18} \mathrm{O}$, which implies that the typical ${ }^{13} \mathrm{CO}$ emission is usually not as optically thick as suggested by this one source. The disk around 2MASS J11095340-7634255, which is bright in ${ }^{13} \mathrm{CO}$, has a $\mathrm{C}^{18} \mathrm{O}$ flux upper limit that is 5-7 times fainter, consistent with the ${ }^{13} \mathrm{CO}$ being optically thin.

For a more universal assessment, we stack the ${ }^{13} \mathrm{CO}$ and $\mathrm{C}^{18} \mathrm{O}$ spectral images for all ${ }^{13} \mathrm{CO}$ detections (excluding 2MASS J11100010-7634578), applying the same approach as described in Section 3.1 and weighting by noise rather than noise squared. The mean signals are $574 \pm 31 \mathrm{mJy} \mathrm{km} \mathrm{s}^{-1}$ in ${ }^{13} \mathrm{CO}$ and $68 \pm 16 \mathrm{mJy} \mathrm{km} \mathrm{s}^{-1}$ in $\mathrm{C}^{18} \mathrm{O}$, which is consistent with most ${ }^{13} \mathrm{CO}$ emission being optically thin. The stacked image of 75 sources that individually lack ${ }^{13} \mathrm{CO}$ emission yields a noiseweighted average line flux of $70 \pm 15 \mathrm{mJy} \mathrm{km} \mathrm{s}^{-1}$ in ${ }^{13} \mathrm{CO}$ and $27 \pm 8 \mathrm{mJy} \mathrm{km} \mathrm{s}^{-1}$ in $\mathrm{C}^{18} \mathrm{O}$. The images of ${ }^{13} \mathrm{CO}$ nondetections, 

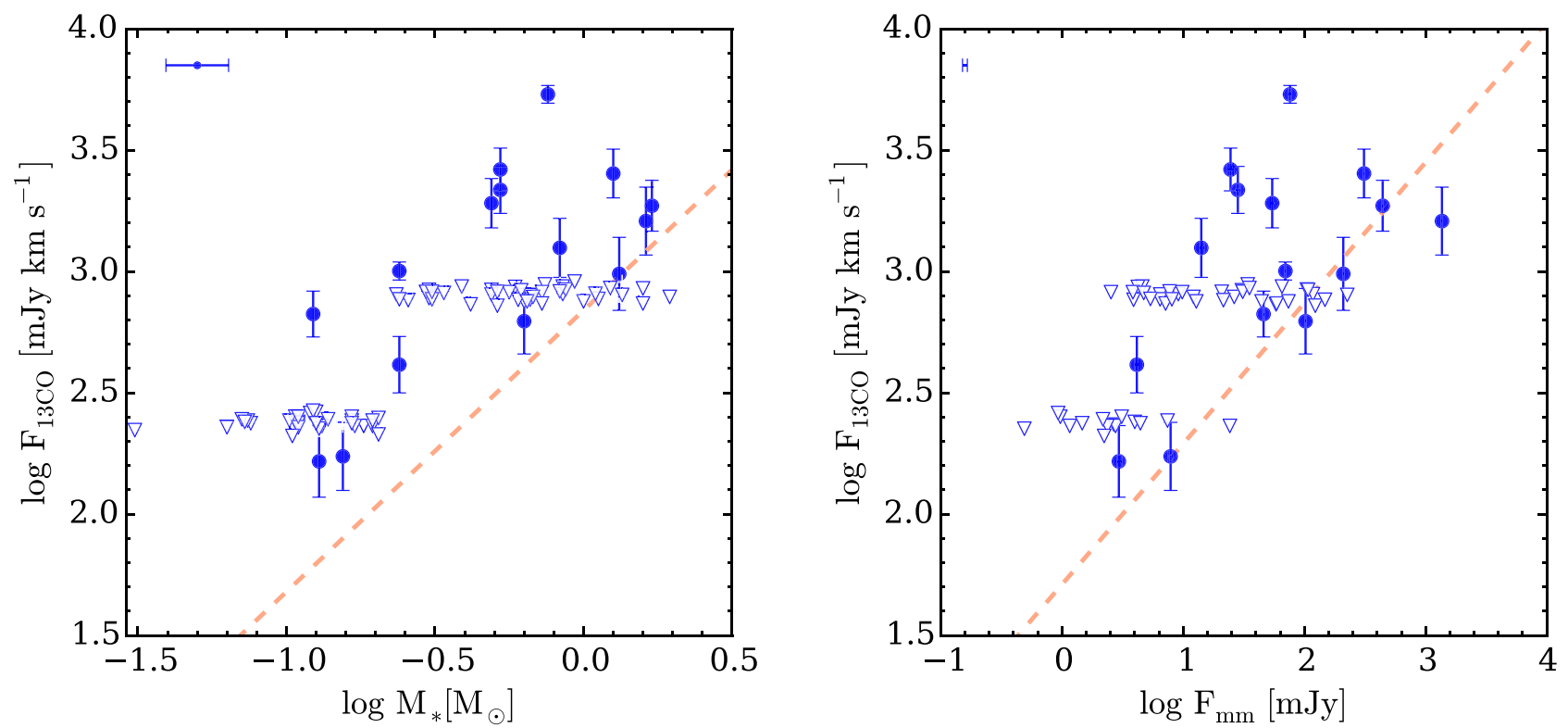

Figure 6. Left: ${ }^{13} \mathrm{CO}$ flux as a function of stellar mass. Right: ${ }^{13} \mathrm{CO}$ flux as a function of $887 \mu \mathrm{m}$ continuum flux density for continuum detections. The ${ }^{13} \mathrm{CO}$ detections are shown as filled circles, while the $3 \sigma$ upper limits are shown as triangles for ${ }^{13} \mathrm{CO}$ nondetections. The best-fit results from Bayesian analysis (dashed lines) are calculated using the measured fluxes and uncertainties for all points, including the nondetections (shown as $3 \sigma$ upper limits only for visualization). The best fits therefore appear lower than the measured data points on the plot and are consistent with results of stacked emission from the nondetections. The median error bar in $\log \left(M_{*}\right)$ and $\log \left(F_{\mathrm{mm}}\right)$ is shown in the upper left corner in each panel.

stacked separately in the Hot and Cool samples, yield fluxes of $59 \pm 15 \mathrm{mJy} \mathrm{km} \mathrm{s}^{-1}$ and $31 \pm 13 \mathrm{mJy} \mathrm{km} \mathrm{s}^{-1}$, respectively. The stacking results confirm the analysis in Section 3.3 that some weak detections may be just beyond our detection limits. The stacked ${ }^{13} \mathrm{CO}$ images for ${ }^{13} \mathrm{CO}$ detections and nondetections are shown in Figure 4.

\subsection{Correlations of Star and Disk Properties with CO Line Fluxes}

The gas mass should depend on the initial mass of the disk, which is expected to vary with stellar mass, and on the massloss rate from the disk due to viscous accretion onto the star and to mass loss in photoevaporative and MHD winds (e.g., Hartmann et al. 1998; Alexander et al. 2014; Armitage 2015). In the viscous accretion disk, the viscous accretion rate should scale with the gas mass. As a consequence, if the ${ }^{13} \mathrm{CO}$ emission is an accurate diagnostic of the gas mass, then we should expect the ${ }^{13} \mathrm{CO}$ flux ${ }^{12}$ to correlate with the dust mass (if dust traces disk mass), the mass of the central star, and the accretion rate onto the star. In this subsection, we investigate whether any of these parameters are correlated with the ${ }^{13} \mathrm{CO}$ line fluxes.

In this analysis, continuum flux densities and stellar masses ${ }^{13}$ of our sample are adopted from Pascucci et al. (2016). The stellar masses were calculated based on evolutionary tracks from nonmagnetic models of Feiden (2016) and Baraffe et al. (2015). For multiple-star systems, the adopted stellar mass is the mass of the primary, following Pascucci et al. (2016). Accretion rates are obtained from Manara et al. (2016a, 2017) and are adjusted for updated luminosities and stellar radii,

\footnotetext{
12 The fluxes may be multiplied by $4 \pi d^{2}$ to convert into luminosity.

13 The stellar masses were calculated assuming a distance of $160 \mathrm{pc}$, but are similar to the masses that would be calculated for the updated $188 \mathrm{pc}$ distance.
}

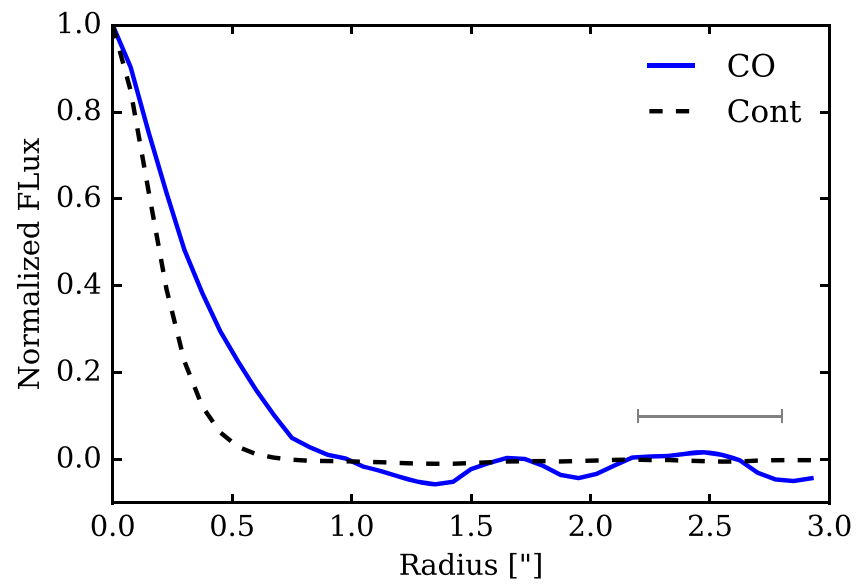

Figure 7. Azimuthally averaged ${ }^{13} \mathrm{CO} J=3-2$ and continuum emission normalized to the peak. The typical beam size is shown in the lower right corner.

which are increased for the adopted distance, and for the masses used by Pascucci et al. (2016).

Figure 5 (left) shows the dust emission versus stellar mass, highlighting the ${ }^{13} \mathrm{CO}$ and $\mathrm{C}^{18} \mathrm{O}$ detections. Overall, sources with $\mathrm{CO}$ emission are located over a wide parameter space in both stellar mass and continuum flux. Within any single stellar mass bin, $\mathrm{CO}$ detections are more likely from sources with strong continuum emission. The strongest continuum emitters in both the Hot and Cool samples are also detected in ${ }^{13} \mathrm{CO}$. When accounting for both significant and tentative detections, the deeper Cool sample has a similar ${ }^{13} \mathrm{CO}$ detection rate to the Hot sample. However, if we consider only the significant detections, nearly $75 \%$ come from the Hot sample, which again suggests that higher-mass stars tend to have brighter $\mathrm{CO}$ emission.

Figure 6 demonstrates the correlation of ${ }^{13} \mathrm{CO}$ flux with respect to stellar mass. The best-fit linear relationship between the $\log$ of the stellar mass and the $\log$ of ${ }^{13} \mathrm{CO}$ flux is 
$\log \left(F_{13 \mathrm{CO}} / \mathrm{mJy}\right)=1.16( \pm 0.13) \times \log \left(M_{*} / M_{\odot}\right)+2.84( \pm 0.18)$, calculated using a Bayesian analysis, as follows. To use the measured line fluxes and uncertainties in the full sample, we adopt the model as $F_{13 \mathrm{CO}}=\beta \cdot M_{*}^{\alpha}$ with $\alpha$ and $\beta$ sampled uniformly in prior range. The $\chi^{2}$ likelihood function is then used to assess the fit quality, accounting for uncertainties in both $F_{13 \mathrm{CO}}$ and $M_{*}$ and for the intrinsic scatter in the relationship. The posterior parameter space is sampled in a Markov chain Monte Carlo (MCMC) algorithm with emcee (Foreman-Mackey et al. 2013). We refer to corner maps in Appendix $\mathrm{C}$ for the posterior analysis results. The linear relation is then recovered by converting the best-fit model to log space. For the nondetections, we use the extracted fluxes and uncertainties instead of 3 times the uncertainty upper limits (see also discussion in Mohanty et al. 2013).

In an alternative approach, the fit is constrained by $3 \sigma$ upper limits rather than the measured data points and uncertainties. In this case, the Bayesian linear regression method Linmix ${ }^{14}$ (Kelly 2007) yields a best fit with a slope of $1.14 \pm 0.27$ and a lower intercept of $2.50 \pm 0.20$. However, because the stacking demonstrates that the upper limits include some signal, we prefer using the full information. The slope is consistent and thus robust in both methods, but the intercept differs owing to different treatment in upper limits and likelihood function.

In these fits, ${ }^{15}$ the power law of 1.16 between stellar mass and ${ }^{13} \mathrm{CO}$ flux is shallower than the power law of 1.9-2.1 between stellar mass and continuum flux from Pascucci et al. (2016). ${ }^{16}$ The relationship between ${ }^{13} \mathrm{CO}$ flux and stellar mass may be steeper if higher sensitivity were achieved, especially for disks around lowmass stars. Since $80 \%$ of our sample is not detected in ${ }^{13} \mathrm{CO}$, more sensitive observations, especially for the low-mass end, are needed to better constrain this relation. The slope between CO flux and stellar mass may also be shallower because the ${ }^{13} \mathrm{CO}$ emission is more likely to be optically thick in more massive disks. The treatment of binarity likely introduces some additional error that is not accounted for in our analysis, since the appropriate mass may be the total system mass for circumbinary disks, or the mass of the secondary if the disk is around the less massive component.

The same Bayesian analysis applied to $887 \mu \mathrm{m}$ continuum fluxes, adopted from Pascucci et al. (2016), and ${ }^{13} \mathrm{CO}$ fluxes for sources detected in continuum yields a best-fit result, $\log \left(F_{13 \mathrm{CO}} / \mathrm{mJy}\right)=0.58( \pm 0.08) \times \log \left(F_{\mathrm{mm}} / \mathrm{mJy}\right)+1.71( \pm 0.16)$.

The right panel of Figure 5 identifies the $\mathrm{CO}$ detections in our sample in a plot of accretion rate versus stellar mass. The only detection in both ${ }^{13} \mathrm{CO}$ and $\mathrm{C}^{18} \mathrm{O}$ lines, 2MASS $\mathrm{J} 11100010-7634578$, is indeed a strong accretor. However, in any

\footnotetext{
14 From https://github.com/jmeyers314/linmix.

15 In these fitting methods, the best-fit results and uncertainties are likely dependent on the assumption of Gaussian scatter and prior distribution in the Bayesian approach, especially when a high fraction of data are upper limits. We therefore adopt a third fitting approach-the cenken routine in the R NADA package (Feigelson \& Babu 2012), which uses the nonparametric AkritasThiel-Sen estimator (Akritas \& Bershady 1996) and makes no initial assumption about the data distribution - to test the reliability of our results. This linear regression method including the censored data information but leaving out the measurement errors provides a fitting result consistent with parametric modeling with slope and intercept of 1.07 and 2.58, respectively.

16 The correlation in stellar mass and continuum flux was calculated using the method of Kelly (2007). We also fit the stellar mass and continuum flux relationship with model $F_{\text {cont }}=\beta \cdot M_{*}^{\alpha}$, which yields a slope of 1.45 and an intercept of 1.87. The fitted correlations in stellar mass and continuum flux from the two methods are reasonably consistent, when only $30 \%$ of data are nondetections. As noted in Linmix, when there is a nondetection, the maximum-likelihood estimate including upper limits may not be valid. When a large fraction of data points are censored, the resulting correlation may not represent the true relation.
}

stellar mass bin, accretion rates of ${ }^{13} \mathrm{CO}$ detections are within the range of accretion rates of ${ }^{13} \mathrm{CO}$ nondetections. A KolmogorovSmirnov two-sample test ${ }^{17}$ of accretion rates in $\mathrm{CO}$ detections and CO nondetections yields a $74 \%$ probability, indicating statistically similar parent distributions. The same Bayesian analysis applied to stellar accretion rate and CO flux yields a slope of only 0.2. From the full sample analysis, there is at most a weak correlation between the gas emission from the disk and the accretion rate. The lack of a correlation is also consistent with the analysis of gas mass, measured from ${ }^{13} \mathrm{CO}$ and $\mathrm{C}^{18} \mathrm{O}$ emission, and accretion rate for disks in Lupus (Manara et al. 2016b).

\subsection{Morphology of CO Emission}

The $887 \mu \mathrm{m}$ continuum images and ${ }^{13} \mathrm{CO}$ intensity maps for each detection are shown in Figure 10. The radial profiles in the stacked continuum and ${ }^{13} \mathrm{CO}$ intensity maps from the full sample, as shown in Figure 7, indicate that ${ }^{13} \mathrm{CO}$ emission is slightly more extended than dust emission, with a radial profile FWHM $30 \%$ wider in ${ }^{13} \mathrm{CO}$ emission. Similarly, many well-known disks, such as HD 163296, V4046 Sgr, and Elias 2-27, have ${ }^{13} \mathrm{CO}$ emission that is 2-3 times more extended than the submillimeter dust emission (e.g., Isella et al. 2007; Rosenfeld et al. 2013; Pérez et al. 2016). The ${ }^{13} \mathrm{CO}$ emission for the tentative detections is typically unresolved in our beam with FWHM of $\sim 0$ !" 6 . The brightest ${ }^{13} \mathrm{CO}$ disk, 2MASS J11095340-7634255, has emission on $2^{\prime \prime}$ scales and would be a factor of two fainter if baselines shorter than $40 \mathrm{k} \lambda$ were excluded.

The ${ }^{13} \mathrm{CO}$ gas emission is spatially resolved in two transition disks, 2MASS J10581677-7717170 and 2MASS J111737007704381 (see also Section 4.4). A lower gas surface density inside the dust cavity is often found from high-resolution observations in transition disks (Bruderer et al. 2014; Zhang et al. 2014; Perez et al. 2015; van der Marel et al. 2015, 2016). In 2MASS J10581677-7717170, which shows an inner dust cavity from submillimeter imaging (Pascucci et al. 2016), the ${ }^{13} \mathrm{CO}$ emission is stronger in the dust ring than in the inner dust cavity, which may be caused by either a gas density drop (van der Marel et al. 2015) or insufficient sensitivity. The ${ }^{13} \mathrm{CO}$ emission is also azimuthally asymmetric, with an emission deficit to the west of the disk and $20 \%-50 \%$ higher flux in the north peak. Even though the dust cavity is not resolved in 2MASS J11173700-7704381, the deficit of ${ }^{13} \mathrm{CO}$ emission is observed in the inner disk. Two emission clumps with peak fluxes differing by $30 \%$ are also seen in the NESW direction. However, due to the limited sensitivity, gas structures presented here may not fully represent the gas distribution in these systems. Higher-resolution and more sensitive observations are therefore needed to recover the gas distribution in detail in these transition disks.

The ${ }^{13} \mathrm{CO}$ moment 1 map (velocity map) and line spectrum for each detection are also shown in Figure 10. Throughout the Cha I sample, two stars, 2MASS J11004022-7619280 and 2MASS J11095340-7634255, show clear double-peaked line profiles in the ${ }^{13} \mathrm{CO}$ spectrum, with distinguishable red- and blueshifted components in velocity maps that indicate Keplerian rotating disks. The narrow velocity range $\left(3.5-6 \mathrm{~km} \mathrm{~s}^{-1}\right)$ in 2MASS J11004022-7619280 suggests that the disk is viewed nearly face-on. Another source, 2MASS J11100010-7634578, has azimuthally asymmetric ${ }^{13} \mathrm{CO}$ emission, with possible absorption at one location that may confuse the detection of

\footnotetext{
17 http://docs.scipy.org/doc/scipy-0.14.0/reference/stats.html
} 
Keplerian rotation (see Appendix B.1.1). Other sources probably lack sufficient $\mathrm{S} / \mathrm{N}$ to display the pattern of Keplerian rotation.

\subsection{CO Emission from Transition Disks}

Transition disks have cavities in their dust distributions, as identified from a deficit of mid-IR emission and from highresolution images of submillimeter continuum emission (see reviews by Williams \& Cieza 2011; Espaillat et al. 2014). Nine disks in our sample were classified as likely transition disks, of which eight candidates were identified indirectly from their mid-IR spectral energy distributions (Kim et al. 2009; Manoj et al. 2011) and one transition disk was identified directly from submillimeter imaging (Pascucci et al. 2016). Four of these transition disks, 2MASS J10581677-7717170, 2MASS J11083905-7716042, 2MASS J11173700-7704381, and the $\epsilon$ Cha member 2MASS $\mathrm{J} 11183572-7935548$, are detected in ${ }^{13} \mathrm{CO}$ emission (including two that are spatially resolved; see Section 4.3). None of the transition disks are detected with $\mathrm{C}^{18} \mathrm{O}$ emission. The fraction of transition disks (or candidates) with detected ${ }^{13} \mathrm{CO}$ emission (4/9) is higher than the fraction of nontransition disks with ${ }^{13} \mathrm{CO}$ detections $(13 / 84)$.

In the comparison sample of Lupus (see also Section 6.1), 12 disks were classified as transition disk candidates from mid-IR spectroscopy, with six confirmed by submillimeter dust imaging (Ansdell et al. 2016). Most (11/12) transition disks in Lupus were detected in ${ }^{13} \mathrm{CO}$, and six of them were also detected in $\mathrm{C}^{18} \mathrm{O}$ (Ansdell et al. 2016). In both the Cha I and the Lupus sample, the ${ }^{13} \mathrm{CO}$ detection rate is higher in the transition disk sample than in the full sample.

\section{CO Gas Masses of Protoplanetary Disks in Cha I}

In this section, we first describe our methods to convert the ${ }^{13} \mathrm{CO}$ and $\mathrm{C}^{18} \mathrm{O}$ line luminosities and upper limits into a gas mass for disks in our sample. We then discuss the range of gas masses and inferred gas-to-dust ratios, as well as the correlation of gas mass with stellar mass. These conversions provided by disk models may severely underestimate the total gas mass in the disk. Large uncertainties in the $\mathrm{CO}$-to- $\mathrm{H}_{2}$ ratio and the gas temperature structure are discussed in detail in Section 6.2.

Much of this analysis uses the stacked fluxes and the best-fit line between the stellar mass and ${ }^{13} \mathrm{CO}$ flux to describe a typical source in the sample. The correlations and some interpretations may be biased to the few disks that are detected in ${ }^{13} \mathrm{CO}$ emission. The use of stacked fluxes should provide a more accurate estimate of the median source, although even these measurements may be skewed by outliers. A more robust analysis would require a much deeper survey with a high detection rate.

\subsection{Converting Line Luminosities to Gas Mass}

The amount of gas in protoplanetary disks provides important constraints on disk evolution and planet formation. Optically thin tracers are needed to probe down to the disk midplane. If the $\mathrm{CO}$ isotopologues are optically thin, as expected for low-mass disks, the uncertainty of converting a detected $\mathrm{CO}$ flux into disk mass resides in the $[\mathrm{CO}] /\left[\mathrm{H}_{2}\right]$ ratio and isotopic ratios. Corrections for the line excitation and optical depth are also uncertain, especially when only individual lines and transitions are considered.
Physical-chemical models are needed to evaluate these processes and to reduce uncertainties, but it is time-consuming to individualize the models to the specific (and often unknown) properties of each source in a survey. To provide a simple conversion factor from the observed line luminosity to gas disk mass, MvD16 developed hundreds of full thermal-chemical models that include isotope-selective photodissociation and CO freeze-out. The models calculate the expected emission from disks with gas masses from $10^{-5}$ to $10^{-1} M_{\odot}$ and a range of radial and vertical structures. In MvD16, stellar luminosities in models are calculated for $1 L_{\odot}$ for T Tauri stars and $10 L_{\odot}$ for Herbig Ae stars. Most of the ${ }^{13} \mathrm{CO}$ detections in our Cha I sample have stellar luminosities between 0.1 and $1 L_{\odot}$. CO line luminosities are fainter by $\sim 25 \%$ for disk models with $0.1 L_{\odot}$ stellar luminosity compared with $\mathrm{T}$ Tauri models (Miotello et al. 2017), which would lead to gas masses that are larger, but still within the uncertainties. In this analysis, we adopt the T Tauri models with stellar luminosity of $1 L_{\odot}$. Other sources of uncertainty are introduced because the accretion rates in the MvD16 models are higher than measured accretion rates in Cha I (Manara et al. 2016a, 2017), and because the MvD16 models calculate models on full disks and do not consider the complicated physical structures that are likely present in all disks, including inner cavities.

In the low-mass disk regime from MvD16 disk models, $\mathrm{CO}$ line luminosities scale linearly with gas disk masses. Since most of our $\mathrm{CO}$ detections are only detected in ${ }^{13} \mathrm{CO}$ and have low line luminosities, we fit simple functions of disk mass with line luminosity using the median value in each mass bin from MvD16 model grids (see Figure 15). Our fitting functions are slightly different from those of Miotello et al. (2017) in the choice of transition mass and in the treatment of disk inclinations in model grids. The gas mass derived using our fitted functions can be converted to the gas masses of Miotello et al. (2017) by dividing our gas mass by factors of 1.6 for disks with an inclination angle of $10^{\circ}$ and by 1.2 for an inclination angle of $80^{\circ}$, respectively (see details in Appendix D). The measured line luminosities or upper limits are adopted for gas mass estimates in the fitted functions of MvD16 model grids, for the ${ }^{13} \mathrm{CO}$ detections or nondetections in our Cha I sample, respectively. For the sources detected only in ${ }^{13} \mathrm{CO}$, gas masses are calculated from the fitted functions of ${ }^{13} \mathrm{CO}$ line luminosities. Upper and lower limits in gas mass are constrained by ${ }^{13} \mathrm{CO}$ line flux uncertainties and $\mathrm{C}^{18} \mathrm{O}$ upper limits. For the one source detected in both lines, we compare the gas mass and upper/lower mass boundaries from two separate fittings and adopt the final gas mass with a lower limit from $\mathrm{C}^{18} \mathrm{O}$ and an upper limit from ${ }^{13} \mathrm{CO}$. For the ${ }^{13} \mathrm{CO}$ nondetections, the upper limits are adopted from the smaller one of the gas mass upper limits, as estimated from ${ }^{13} \mathrm{CO}$ and $\mathrm{C}^{18} \mathrm{O}$ upper limits of line luminosities. The gas masses and upper/lower limits for our sample are listed in Table 3.

An alternate effort by WB14 used parameterized model grids to estimate gas disk mass. The effects of $\mathrm{CO}$ freeze-out and $\mathrm{CO}$ photodissociation are explored under different surface density distributions, gas temperature profiles, gas disk masses, and disk geometries. The isotope-selective photodissociation is estimated by reducing the $\mathrm{C}^{18} \mathrm{O}$ abundance by a factor of 3 . Tests of a few well-studied disks (e.g., DM Tau, GG Tau) suggest that this reduced $\mathrm{C}^{18} \mathrm{O}$ abundance yields results that are consistent with results from chemical models (Dutrey et al. 1997). To conduct a direct comparison of the two models, we 

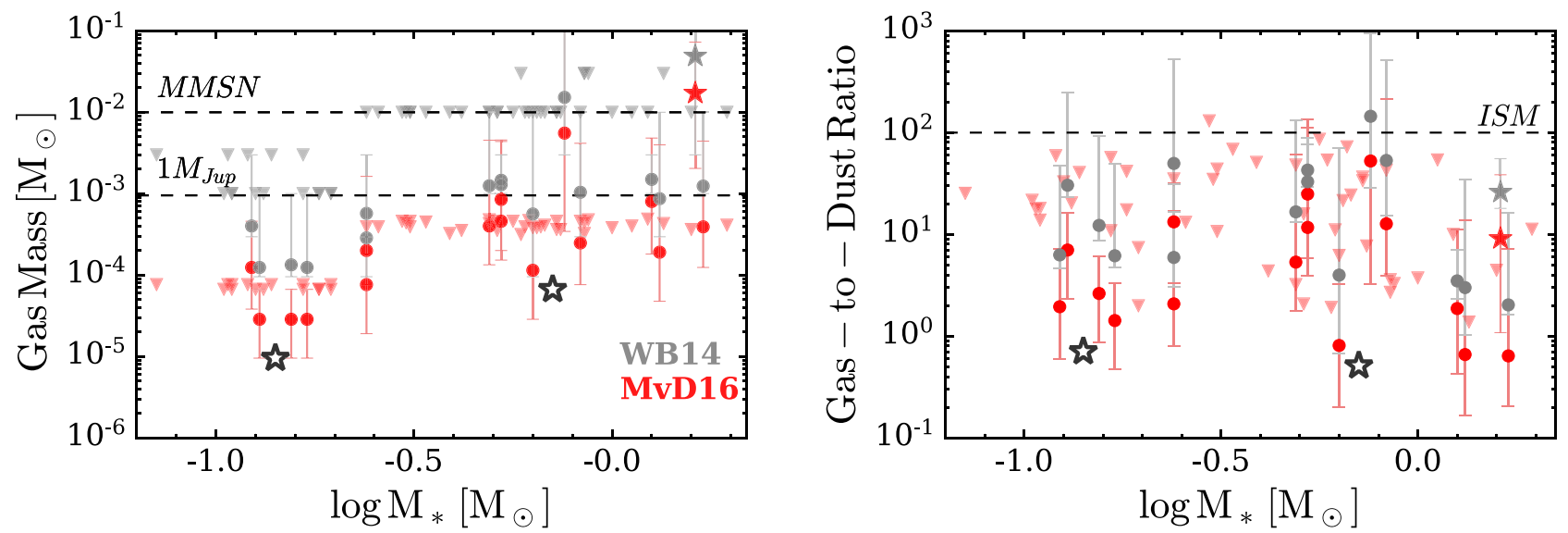

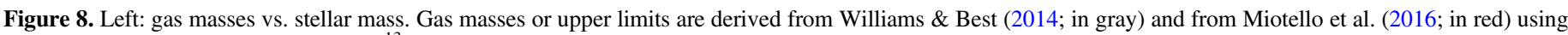

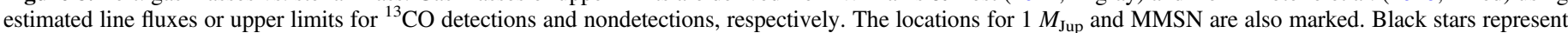

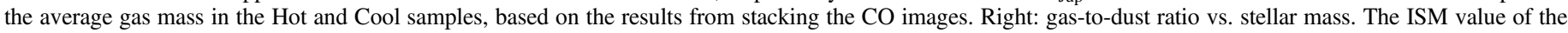
gas-to-dust ratio is labeled out. The only detection in both lines, 2MASS J11100010-7634578, is marked with a filled star.

derive the gas mass from WB14 model grids by adopting the same approach described in detail in Ansdell et al. (2016). We search for model grids of simulated line luminosities within uncertainties of our observed ${ }^{13} \mathrm{CO}$ and $\mathrm{C}^{18} \mathrm{O}$ line luminosities or constrained by upper limits. Gas masses for ${ }^{13} \mathrm{CO}$ detections are adopted as the mean value of the $\log M_{\text {gas }}$ from the acceptable models to reduce the effects of extremely large or small grid points. The upper $\left(M_{\max }\right)$ and lower $\left(M_{\min }\right)$ boundaries are also set by the maximum and minimum $M_{\text {gas }}$ in the accepted model grids. For sources without detected ${ }^{13} \mathrm{CO}$ emission, only upper limits for gas mass are provided. The gas masses and upper limits derived from WB14 are also listed in Table 3. The high gas mass upper limits derived from WB14 are from disk models with low atmospheric temperatures and rapidly decreasing temperature profiles (e.g., $T_{\text {atm, } 1 \text { au }}=500 \mathrm{~K}$, $q=0.65$ ). If these specific model grids are excluded, then the upper limits on gas mass would not be much higher than gas masses inferred from ${ }^{13} \mathrm{CO}$ detections.

\subsection{Gas Masses and Gas-to-dust Ratios}

The derived gas masses and upper limits for continuum detections in our Cha I sample are shown in the left panel of Figure 8, from both WB14 and MvD16 models. Gas masses inferred from $\mathrm{CO}$ detections are very low, spanning from 0.03 to 1 $M_{\text {Jup }}\left(10-300 M_{\text {Earth }}\right)$. The two exceptions, 2MASS J110953407634255 and J11100010-7634578, have gas masses similar to the Minimum Mass Solar Nebula (MMSN, $0.01 M_{\odot}$ ), the mass of materials required to form the solar planetary system (Kusaka et al. 1970; Weidenschilling 1977). We also stack the Hot and Cool sample separately in ${ }^{13} \mathrm{CO}$ and calculate the gas mass in MvD16 fitted functions. The stacked line fluxes correspond to gas masses of $\sim 0.07 M_{\text {Jup }}\left(22 M_{\text {Earth }}\right.$ ) for the Hot sample and $\sim 0.01$ $M_{\text {Jup }}$ ( $3 M_{\text {Earth }}$ ) for the Cool sample.

Gas masses from MvD16 models are generally lower than from WB14 model grids, mainly due to a wider gas temperature range adopted in WB14 models (as discussed by MvD16). If additional depletion factors in the $\mathrm{C}^{18} \mathrm{O}$ abundance for lower-mass stars are included in WB14 models, as suggested by the models of MvD16, then the parameterized models would yield even higher gas masses.

The inferred gas-to-dust ratios are shown in the right panel of Figure 8, with the dust mass calculated by Pascucci et al. (2016) assuming a fixed dust temperature of $20 \mathrm{~K}$ and then scaled to the 188 pc Gaia distance. Since gas mass upper limits derived from WB14 are biased toward one set of disk models (see discussion in Section 5.1), gas-to-dust ratios based on WB14 gas mass are only shown for $\mathrm{CO}$ detections. The gas-to-dust ratios are spread over two orders of magnitude for sources detected with $\mathrm{CO}$ emission, and most sources have lower ratios than the typical ISM value ( $\sim 100)$. The median value of the gas-to-dust ratio is $\sim 4$ when the gas mass is evaluated from MvD16 and $\sim 15$ when evaluated from WB14. In most CO nondetections, gas-to-dust ratios are also lower than the typical ISM value. The stacked gas masses in the Hot and Cool samples, when applied with the mean dust mass in each sample, yield an average gas-to-dust ratio of $\sim 1$, two orders of magnitude lower than the ISM value.

The choice of dust temperature affects the dust mass measurements (see discussion in Andrews et al. 2013; van der Plas et al. 2016; Pascucci et al. 2016). If $T_{\text {dust }}$ scales with $L_{*}$, we would obtain lower gas-to-dust ratios in very low mass stars than when a constant $T_{\text {dust }}=20 \mathrm{~K}$ is assumed, as cooler temperature leads to higher dust mass. However, $T_{\text {dust }}$ is independent of $L_{*}$, if dust disk size scales linearly with stellar mass (Hendler et al. 2017). The dependence of disk radii on stellar mass is not yet well quantified in unbiased samples. The low gas masses and low gasto-dust ratios are consistent with the results from a small sample in Taurus (Williams \& Best 2014) and a nearly complete Class II disk sample in Lupus (Ansdell et al. 2016; Miotello et al. 2017). We will discuss the implications of the low gas masses and gas-todust ratios in Section 6.2.

While the scaling relation between dust disk mass and stellar mass has been measured in multiple star-forming regions (Andrews et al. 2013; Mohanty et al. 2013; Ansdell et al. 2016; Barenfeld et al. 2016; Pascucci et al. 2016), the scaling relation between gas disk mass and stellar mass is less understood and only limited to the Lupus clouds (Miotello et al. 2017).

Due to the high rate of $\mathrm{CO}$ nondetections in our Cha I sample, we establish an empirical correlation between the gas mass and the stellar mass based on the relation of CO line flux with stellar mass and the conversion of $\mathrm{CO}$ line flux to gas mass. We adopt the best fit of ${ }^{13} \mathrm{CO}$ line flux with stellar mass in Section 4.2 and then convert the ${ }^{13} \mathrm{CO}$ line fluxes to gas masses based on the fitted functions of the median ${ }^{13} \mathrm{CO}$ line luminosity with gas mass from MvD16 models in Section 5.1. The best-fit gas mass and stellar mass relation is given as $\log \left(M_{\text {gas }} / M_{\mathrm{Jup}}\right)$ 

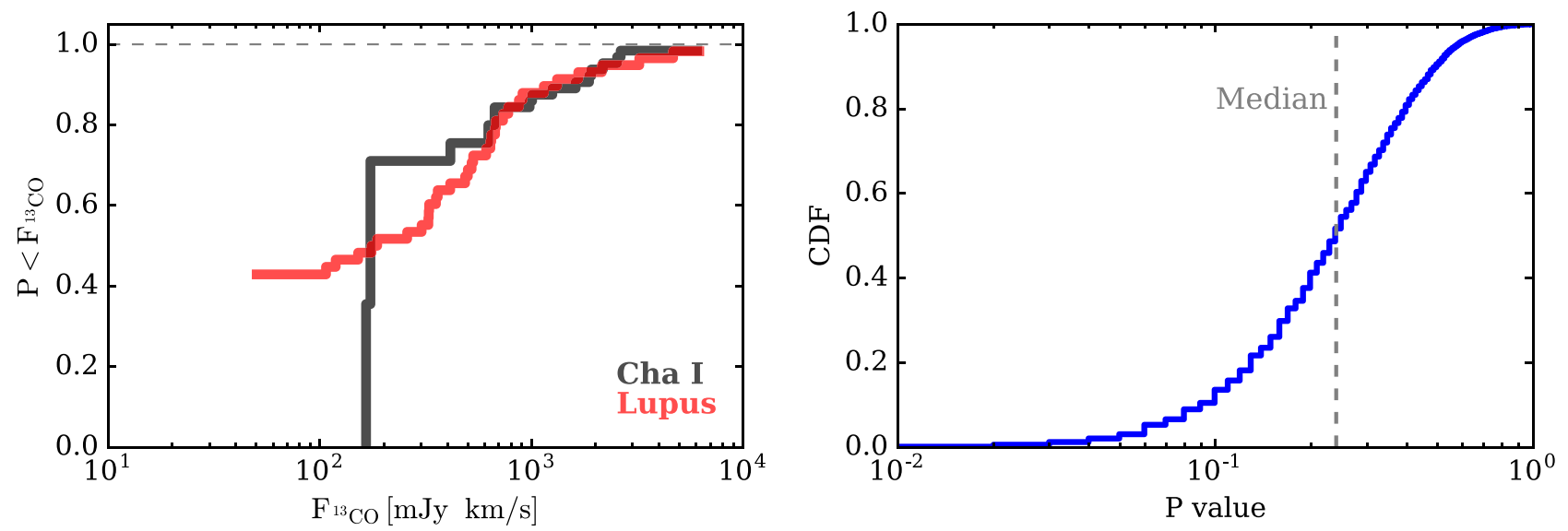

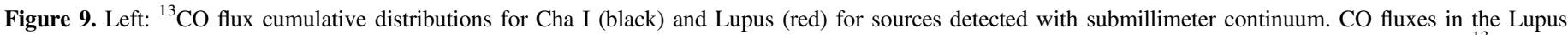

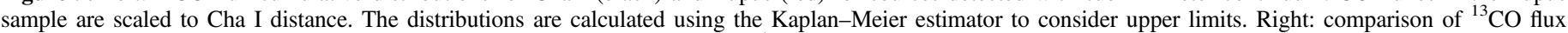

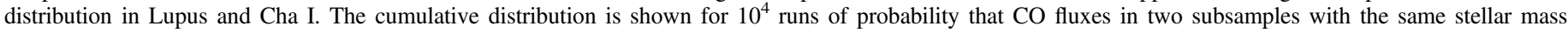
distribution are drawn from the same parent distribution.

$=1.27( \pm 0.14) \times \log \left(M_{*} / M_{\odot}\right)-0.76( \pm 0.11)$. This fit only provides an average gas mass in a given stellar mass bin and is highly dependent on the robustness of the relation of ${ }^{13} \mathrm{CO}$ flux and stellar mass. The real relation may be steeper, since ${ }^{13} \mathrm{CO}$ emission is more likely to be optically thick in more massive disks and lines from smaller disks are likely to be fainter. Miotello et al. (2017) found a power-law index of 0.63 between disk gas mass and stellar mass in Lupus clouds with 34 detections of ${ }^{13} \mathrm{CO}$ emission and $10 \mathrm{C}^{18} \mathrm{O}$ detections, with the rest being nondetection upper limits. More $\mathrm{CO}$ detections from highsensitivity data sets and more accurate gas mass estimates are needed to evaluate the robustness of the established correlation between gas mass and stellar mass.

\section{Discussion}

Submillimeter continuum surveys of protoplanetary disks have all found that stellar masses and dust disk masses are strongly correlated, though with large scatter (see review by Williams \& Cieza 2011). The disks in the 1-3 Myr old regions of Taurus, Lupus, and Cha I separately lead to indistinguishable relationships between the dust disk mass and stellar mass (Andrews et al. 2013; Ansdell et al. 2016; Pascucci et al. 2016). In this section, we compare the $\mathrm{CO}$ gas properties of Cha I found here with those obtained in the ALMA survey of Lupus disks, which had a similar experimental design that targeted ${ }^{13} \mathrm{CO}$ and $\mathrm{C}^{18} \mathrm{O}$ emission. ${ }^{18} \mathrm{We}$ then discuss the implications for the weak $\mathrm{CO}$ emission detected from both Cha I and Lupus.

\subsection{Comparison to $\mathrm{CO}$ Emission from the Lupus Molecular Cloud}

The ALMA survey of Lupus was implemented with a strategy similar to our Cha I survey. The Lupus sample was split into M dwarfs, with a sensitivity comparable to our Cool sample in Cha I (M3-M8), and AFGK stars, with a sensitivity 2-3 times deeper than the Hot sample (K0-M3) in Cha I. Twenty sources in Lupus lack optical/near-IR spectral types, are likely highly obscured or very low mass objects, and are excluded from all comparisons presented here. Stellar and CO luminosities of Lupus objects

\footnotetext{
18 The ALMA continuum survey of the Upper Sco Association (Barenfeld et al. 2016) observed the ${ }^{12} \mathrm{CO}$ line, which probes the disk surface area instead of bulk gas mass.
}

are adjusted to the updated Gaia distance of $157 \pm 7 \mathrm{pc}$ (see Appendix A). The average age of stars with disks in Lupus ( $\sim 2 \mathrm{Myr})$ is indistinguishable from the average age of those in Cha I.

The submillimeter continuum detection rate is higher in Lupus (58/69) than in Cha I (65/92). Pascucci et al. (2016) found that a single $M_{\text {dust }}-M_{*}$ relation can explain the dust disk masses in both clusters within measurement uncertainties, with a similarity that is improved with the updated distances. The submillimeter dust emission suggests that Lupus and Cha I are in the same stage of disk evolution. In contrast, the ${ }^{13} \mathrm{CO}$ detection rate is much lower in Cha I $(17 \% \text {, or } 15 / 91)^{19}$ than in Lupus $(48 \%$, or $33 / 69)$. Because most of these detections are near the sensitivity limits, such a large difference may be explained by the differences in depth and sample. When the Lupus sample is scaled to the Cha I distance and assigned the sensitivity of our survey, the ${ }^{13} \mathrm{CO}$ detection rate in Lupus would decrease to $24 \%$, similar to our detection rate for Cha I. When separated into stars of spectral type $\mathrm{K}, \mathrm{M} 0-\mathrm{M} 3$, and M4-M6, the detection rate in each subsample is only slightly higher in Lupus than in Cha I, which may be explained by the different methodologies.

The ${ }^{13} \mathrm{CO}$ flux cumulative distributions for Cha I and Lupus, scaled to Cha I distance, are shown in the left panel of Figure 9. The distributions are calculated using the Kaplan-Meier estimator in the R NADA package to include $3 \sigma$ upper limits. The mean ${ }^{13} \mathrm{CO}$ flux is slightly higher in Lupus than in Cha I. The two distributions are generally consistent at the higher flux end. The large discrepancy in the low flux regime is mainly due to different sensitivities in the two regions. We also perform the two-sample test with the cendiff routine, including censored data in the NADA package, which estimates the probability of two samples sharing the same parent distribution. We first constrain the stellar mass distribution by assigning the same number of sources in each stellar mass bin in both clusters, following the same approach as in Andrews et al. (2013) and Ansdell et al. (2016). A two-sample test of ${ }^{13} \mathrm{CO}$ flux with censored data in the two subsamples with the same stellar mass distribution is then calculated. The cumulative distribution of statistical results $\left(P\right.$ value) from $10^{4}$ iterations is shown in the

\footnotetext{
19 2MASS J11183572-7935548, a likely member of $\epsilon$ Cha, and 2MASS J11075792-7738449, with offset ${ }^{13} \mathrm{CO}$ emission from extended nebulosity, are excluded.
} 
right panel of Figure 9, with the median $P$ value of 0.25 , indicating that the ${ }^{13} \mathrm{CO}$ flux distributions in Cha I and Lupus are statistically indistinguishable.

However, the conclusions from this comparison would change if the lower value of upper limits for ${ }^{13} \mathrm{CO}$ nondetections from the 0 . 3 radius aperture were adopted. The ${ }^{13} \mathrm{CO}$ cumulative distributions for the two samples would be consistent at high fluxes, but the distribution in the Cha I sample would flatten out at low fluxes. The same two-sample test in $10^{4}$ iterations results in a median $P$ value of 0.003 , which would suggest ruling out the hypothesis that the disk masses from both regions are statistically indistinguishable.

Because of the high fraction of nondetections in both samples, the differences in the beam sizes, sensitivities, and extraction methods prevent any firm conclusion on the similarity of CO gas properties in the disks in Lupus and Cha I.

\subsection{CO Depletion and Implications for Planet Formation}

$\mathrm{CO}$ line emission has been previously found to be fainter than expected from the dust emission, assuming the canonical ISM gas-to-dust and $[\mathrm{CO}] /\left[\mathrm{H}_{2}\right]$ ratios (e.g., Dutrey et al. 2003; Chapillon et al. 2008; Rodriguez et al. 2015; Pericaud et al. 2016). This conclusion, from small and biased samples, was recently strengthened in a large and unbiased sample of disks in the Lupus star-forming region (Ansdell et al. 2016; Miotello et al. 2017) and is confirmed here for a complete survey of disks from the young (2-3 Myr) Cha I star-forming region. The derived low CO-based gas mass in 2-3 Myr old disks has three possible explanations: (1) the conversion of $\mathrm{CO}$ line flux to $\mathrm{CO}$ gas mass is incorrect, (2) the total gas mass in these disks is very low, or (3) the $\mathrm{C}$ depletion into $\mathrm{CO}$ and/or complex ices is underestimated, thereby altering the $\mathrm{CO}-$ to $-\mathrm{H}_{2}$ ratio. We discuss each of these possibilities in the following subsections.

\subsubsection{Conversion of CO Line Flux to CO Gas Mass}

The conversion of $\mathrm{CO}$ line flux to $\mathrm{CO}$ gas mass requires the application of physical-chemical models, along with the canonical assumption that the CO-to- $\mathrm{H}_{2}$ ratio is $\sim 10^{-4}$. Since each individual disk cannot be modeled, we rely on results from grids of disk models (WB14 and MvD16) that were calculated over ranges of disk and stellar properties. As discussed in Section 5.1, these grids are not complete. Relative to our sample, the stellar mass and mass accretion rates in these models are high. Mini-grids in Miotello et al. (2017) suggest that these results are robust to difference in stellar mass (stellar luminosity), though the uncertainties induced by changing the accretion rates are not explored.

The CO gas masses obtained from conversions using the WB14 and MvD16 grids are both very low, despite very different modeling approaches and implementation of isotopicselective photodissociation. However, these conversions should be confirmed with further tests using independent disk models and dedicated analyses of disks with well-known structures. The uncertainties in the disk models include the temperature structure of the gas and the physical structure of the disk. Model grids cannot be expected to account for the many disk structures that must exist in our sample, and in any case our knowledge of these structures is very limited. If the gas surface density peaks sharply near the star, most of the gas will be optically thick and will not contribute significantly to the measured fluxes (Yu et al. 2016, 2017). Observations of multiple CO lines at high spatial resolution could help to resolve this potential error.

\subsubsection{Very Low Total Gas Masses}

If gas masses are very low, the disk dispersal timescales for these disks would be implausibly short. Under the assumption that the ${ }^{13} \mathrm{CO}$ emission is accurately converted into a disk mass, a typical $0.7 M_{\odot}$ star in Cha I would have a disk gas mass of $\sim 10^{-4} M_{\odot}$. With a typical accretion rate of $2 \times 10^{-9} M_{\odot} \mathrm{yr}^{-1}$ (Manara et al. 2016a, 2017), disks would disperse within $\sim 10^{5}$ yr a very short time relative to the current age of $\sim 2 \mathrm{Myr}$ and the average disk dispersal timescale of 2-5 Myr (e.g., Haisch et al. 2001; Hernández et al. 2008; Fedele et al. 2010). Most gaseous disks would have already disappeared.

However, if the conversions between $\mathrm{CO}$ emission and $\mathrm{CO}$ gas mass are somehow correct, and the $\mathrm{C}$ is not depleted, then gas masses for the $2 \mathrm{Myr}$ old disks in Cha I and in Lupus are very low and insufficient to form a $\sim 1 M_{\text {Jup }}$ giant planet. An early start and completion of giant planet formation would be required to explain the abundance of giant planets in the observed planet population (Cumming et al. 2008; Winn \& Fabrycky 2015). The low gas-to-dust ratios implied by the low gas masses may facilitate the formation of planetesimals through either gravitational collapse or streaming instability (Youdin \& Shu 2002; Youdin \& Goodman 2005; Bai \& Stone 2010). The remaining mass in disks would be sufficient to continue forming super-Earth and Neptune-mass planets, potentially explaining their prevalence around solar-type stars (Howard et al. 2010; Mayor et al. 2011; Petigura et al. 2013; Mulders et al. 2015b).

\subsubsection{CO Depletion into Ices}

These gas masses are also much lower than those obtained from HD emission (Bergin et al. 2013; McClure et al. 2016), although the HD line was only detected for a few sources. The $\mathrm{CO}$ emission may not be an accurate tracer of the gas mass (see also Miotello et al. 2017). A low CO abundance, and therefore weak emission, may result from most $\mathrm{C}$ turning into more complex carbon chains and freezing out into ices. Since the gas mass depends inversely on $[\mathrm{CO}] /\left[\mathrm{H}_{2}\right]$, a higher gas mass would be obtained if the $[\mathrm{CO}] /\left[\mathrm{H}_{2}\right]$ abundance ratio were lower. The few detections of HD emission from disks (with three detections from seven objects in the sample) suggest that the gas mass may be 10 times higher than that inferred from modeling the observed ${ }^{13} \mathrm{CO}$ emission and provides gas-to-dust ratios with median values consistent with 100 (Bergin et al. 2013; McClure et al. 2016). In addition, the relationship between disk mass and mass accretion rate in Lupus supports predictions from viscous accretion, if gas-to-dust ratios are 100 (Manara et al. 2016b); however, modeling results of dust disk masses and accretion rates in Cha I may be more challenging to explain with simple viscous accretion (G. D. Mulders et al. 2017, in preparation).

These results suggest a constant ISM gas-to-dust ratio in disks and in turn imply that gas masses from ${ }^{13} \mathrm{CO}$ are severely underestimated. On the other hand, direct absorption-line measurements indicate a $[\mathrm{CO}] /\left[\mathrm{H}_{2}\right]$ abundance ratio consistent with the ISM in the warm disk surface around RW Aur (France et al. 2014). The CO depletion may vary with both disk radius and disk height. Active carbon chemistry leads to the formation of carbon chains or $\mathrm{CO}_{2}$ that freeze out subsequently to lock up 
the carbon and oxygen elements in the solids and thus lower the $[\mathrm{CO}] /\left[\mathrm{H}_{2}\right]$ abundance ratio (Favre et al. 2013; Du et al. 2015; Kama et al. 2016a, 2016b; Yu et al. 2016; Xu et al. 2017). This interpretation was independently suggested also as the favored explanation for the low ${ }^{13} \mathrm{CO}$ fluxes in Lupus in the recent analysis by Miotello et al. (2017). Additionally, the depletion of oxygen is also suggested from water observations and simulations as freeze-out of volatiles followed by grain growth and settling (Krijt et al. 2016; Du et al. 2017) and has significant effects on hydrocarbon abundances (Bergin et al. 2016).

If carbon and/or oxygen depletion is more severe than is currently estimated in the chemical models, a higher gas mass and gas-to-dust ratio would be recovered. The ISM gas-to-dust ratio can be achieved if $\mathrm{CO}$ abundance is depleted by a factor of 10-100, in which case gas masses in disks around the higher-mass stars would be similar to the MMSN. In this case, measurements of $\mathrm{CO}$ depletion are required to calibrate the CO-based gas mass. A high $\mathrm{C}$ abundance in ices of complex molecules should affect the abundances in any planets that form within these disks, similar to the processes suggested for the $\mathrm{CO}$ and $\mathrm{H}_{2} \mathrm{O}$ ice lines by Öberg et al. (2011).

\section{Conclusions}

In this paper, we present an ALMA survey of ${ }^{13} \mathrm{CO}$ and $\mathrm{C}^{18} \mathrm{O}$ line emission in a large sample (93, complete down to 0.1 $\left.M_{\odot}\right)$ of protoplanetary disks in the nearby $(\sim 188 \mathrm{pc})$ and young ( $\sim 2$ Myr) Chamaeleon I star-forming region. We develop a uniform method to identify detections and measure line fluxes, uncertainties, and upper limits. This method is optimized for analysis of surveys dominated by weak signals and upper limits. The gas masses of these disks are then estimated using the ${ }^{13} \mathrm{CO}$ and $\mathrm{C}^{18} \mathrm{O} J=3-2$ lines to understand how rapidly the disk evolves. Our main conclusions are as follows:

1. We detect ${ }^{13} \mathrm{CO}$ emission from 17 out of 93 sources (15 of 92 sources in Cha I with a disk origin), consisting of 12 significant detections and 5 tentative detections. Only one disk, 2MASS J11100010-7634578, is detected in both ${ }^{13} \mathrm{CO}$ and $\mathrm{C}^{18} \mathrm{O}$ lines. The sources with ${ }^{13} \mathrm{CO}$ detections have a wide spread in stellar mass, submillimeter continuum flux, and accretion rate. The detection rates and line fluxes from stacked observations suggest that in most cases the measured ${ }^{13} \mathrm{CO}$ emission is optically thin on spatial scales of $\sim 0$ ". $5-1$." 0 , although the only source with $\mathrm{C}^{18} \mathrm{O}$ detection is optically thick in ${ }^{13} \mathrm{CO}$ (line flux ratio $\sim 1-2$ ).

2. Gas masses are estimated by adopting the parametric models (WB14) and full-chemical models (MvD16). Even though gas masses derived from WB14 are generally higher than from MvD16, the CO isotopologue emission constrains the $M_{\text {gas }}$ around $1 M_{\text {Jup }}$ in the CO detections. The average gas masses derived from the stacked ${ }^{13} \mathrm{CO}$ line fluxes are $0.07 M_{\mathrm{Jup}}$ and $0.01 M_{\mathrm{Jup}}$ for the Hot and Cool samples, respectively. If these gas masses are correct, then the derived gas-to-dust ratios would be $1-10$, much lower than the standard ISM value of 100. These tiny disk masses inferred from the CO fluxes and chemical model grids imply dispersal timescales of disks through viscous accretion that are implausibly short, as estimated from measured accretion rates in Manara et al. (2016a, 2017).
3. The low gas masses and low gas-to-dust ratios in Cha I, as derived from disk models with $\mathrm{CO}$ isotopologue emission, confirm similar results from a disk survey of the 1-3 Myr Lupus star-forming region. However, whether the disks from Cha I have CO fluxes similar to or weaker than those of the disks in Lupus is uncertain because of observational differences and low detection rates.

4. The conversions of CO flux to $\mathrm{CO}$ gas mass depend on the accuracy of the disk models, which have not been adapted to the full range of stellar luminosities, accretion rates, and disk structures. The gas masses may be severely underestimated if $\mathrm{CO}-$ to- $\mathrm{H}_{2}$ abundance ratio is lower than the ISM value, which may be caused by $\mathrm{C}$ and/or $\mathrm{O}$ depletion and lock-up, or if $\mathrm{CO}$ freeze-out is underestimated. Additional observations of $\mathrm{CO}$ and other carbon-bearing species are needed to gain a comprehensive understanding on CO chemistry and thus the related gas masses.

We appreciate the thorough and constructive comments from the anonymous referee, which served to improve the rigor and clarity of our results. We thank Fred Ciesla, Sebatiaan Krijt, Johan Olofsson, Germano Sacco, and Laszlo Szucs for contributions to the proposal that was awarded for these data. F.L. thanks Ran Wang and Chaoli Zhang for help learning CASA and Zhiyuan Ren and Jinghua Yuan for help with python plotting. F.L. and G.J.H. also thank Emma Yu and Neal Evans for discussion about $\mathrm{C}$ depletion and Uma Gorti for a discussion on gas temperatures. F.L. and G.J.H. are supported by general grant 11473005 awarded by the National Science Foundation of China. I.P. acknowledges support from an NSF Astronomy \& Astrophysics Research Grant (ID: 1515392). C.F.M. gratefully acknowledges an ESA Research Fellowship. The results reported herein benefitted from collaborations and/ or information exchange within NASA's Nexus for Exoplanet System Science (NExSS) research coordination network sponsored by NASA's Science Mission Directorate.

This paper is based on the following ALMA data: ADS/ JAO.ALMA\#2013.1.00437.S. ALMA is a partnership of ESO (representing its member states), NSF (USA), and NINS (Japan), together with NRC (Canada) and NSC and ASIAA (Taiwan), in cooperation with the Republic of Chile. The Joint ALMA Observatory is operated by ESO, AUI/NRAO, and NAOJ. This work has also made use of data from the European Space Agency (ESA) mission Gaia (https://www.cosmos.esa. int/gaia), processed by the Gaia Data Processing and Analysis Consortium (DPAC, https://www.cosmos.esa.int/web/gaia/ $\mathrm{dpac} /$ consortium). Funding for the DPAC has been provided by national institutions, in particular the institutions participating in the Gaia Multilateral Agreement.

\section{Appendix A \\ Distances to the Cha I and Lupus Star-forming Regions}

We calculated a distance of $188 \pm 12 \mathrm{pc}$ to Cha I from the average Gaia DR1 TGAS parallax to Cha I members HD 97048, HD 97300, CV Cha, CR Cha, and DI Cha (Gaia Collaboration et al. 2016). The uncertainty includes a systematic uncertainty in parallax of $\sim 0.3$ mas yr$^{-1}$ and a standard deviation of 0.2 mas yr $^{-1}$ in the measurements and excludes uncertainty introduced by the decision to ignore candidate members HD 93828 and HD 96675. The star HD 93828 is spatially coincident with the projected 
Table 2

Source Properties and Measured CO Fluxes

\begin{tabular}{|c|c|c|c|c|c|c|c|c|}
\hline \multirow{3}{*}{ 2MASS } & \multirow{3}{*}{ SpTy } & \multirow{3}{*}{$\begin{array}{c}\log \left(M_{*}\right) \\
\left(M_{\odot}\right)\end{array}$} & \multirow{3}{*}{$\begin{array}{c}F_{887 \mu \mathrm{m}} \\
(\mathrm{mJy})\end{array}$} & \multicolumn{3}{|c|}{${ }^{13} \mathrm{CO} J=3-2$} & \multicolumn{2}{|c|}{$\mathrm{C}^{18} \mathrm{O} J=3-2$} \\
\hline & & & & \multirow{2}{*}{$\begin{array}{c}\text { Flux } \\
\left(\mathrm{mJy} \mathrm{km} \mathrm{s}^{-1}\right)\end{array}$} & \multicolumn{2}{|c|}{$\mathrm{S} / \mathrm{N}$} & \multirow{2}{*}{$\begin{array}{c}\text { Flux } \\
\left(\mathrm{mJy} \mathrm{km} \mathrm{s}^{-1}\right)\end{array}$} & \multirow{2}{*}{$\begin{array}{l}\mathrm{S} / \mathrm{N} \\
0 ! 3 \\
\end{array}$} \\
\hline & & & & & $0 . " 6$ & $0 . " 3$ & & \\
\hline J10533978-7712338 & M2 & -0.41 & $4.6 \pm 0.79$ & $240 \pm 289$ & 0.8 & 1.5 & $95 \pm 119$ & 0.8 \\
\hline J10555973-7724399 & K7 & -0.13 & $34.1 \pm 1.32$ & $-525 \pm 295$ & -1.8 & -1.6 & $-39 \pm 117$ & -0.3 \\
\hline J10561638-7630530 & M6.5 & -0.96 & $3.99 \pm 0.16$ & $116 \pm 80$ & 1.4 & 2.2 & $-57 \pm 45$ & -1.3 \\
\hline J10563044-7711393 & K7 & -0.07 & $117.58 \pm 1.1$ & $508 \pm 269$ & 1.9 & 1.0 & $144 \pm 134$ & 1.1 \\
\hline J10574219-7659356 & M3 & -0.52 & $9.12 \pm 0.83$ & $265 \pm 269$ & 1.0 & 0.8 & $13 \pm 123$ & 0.1 \\
\hline J10580597-7711501 & M5.5 & -0.96 & $2.68 \pm 0.16$ & $102 \pm 78$ & 1.3 & 0.5 & $71 \pm 43$ & 1.7 \\
\hline J10581677-7717170 & $\mathrm{K} 2$ & 0.1 & $310.18 \pm 1.0$ & $1091 \pm 271$ & 4.0 & 3.5 & $10 \pm 129$ & 0.1 \\
\hline J10590108-7722407 & K7 & -0.07 & $65.34 \pm 1.7$ & $224 \pm 289$ & 0.8 & 0.2 & $111 \pm 125$ & 0.9 \\
\hline J10590699-7701404 & K0 & 0.23 & $442.18 \pm 0.76$ & $1361 \pm 278$ & 4.9 & 5.7 & $17 \pm 118$ & 0.1 \\
\hline J11004022-7619280 & M4 & -0.62 & $69.75 \pm 0.17$ & $602 \pm 82$ & 7.3 & 7.4 & $99 \pm 44$ & 2.3 \\
\hline J11022491-7733357 & $\mathrm{K} 2$ & 0.13 & $225.68 \pm 0.74$ & $740 \pm 267$ & 2.8 & 2.4 & $296 \pm 130$ & 2.3 \\
\hline J11023265-7729129 & M3 & -0.52 & $-0.21 \pm 0.82$ & $466 \pm 280$ & 1.7 & 1.1 & $76 \pm 129$ & 0.6 \\
\hline J11025504-7721508 & M4.5 & -0.74 & $1.16 \pm 0.16$ & $-55 \pm 77$ & -0.7 & -0.4 & $-9 \pm 44$ & -0.2 \\
\hline J11040425-7639328 & M4.5 & -0.74 & $2.77 \pm 0.16$ & $180 \pm 77$ & 2.3 & 2.4 & $10 \pm 49$ & 0.2 \\
\hline J11040909-7627193 & K5 & -0.06 & $104.78 \pm 0.6$ & $-259 \pm 281$ & -0.9 & -1.4 & $-43 \pm 125$ & -0.3 \\
\hline J11044258-7741571 & M4 & -0.62 & $4.15 \pm 0.16$ & $336 \pm 77$ & 4.3 & 4.9 & $45 \pm 43$ & 1.0 \\
\hline J11045701-7715569 & M3 & -0.53 & $2.54 \pm 0.81$ & $284 \pm 274$ & 1.0 & 1.5 & $-124 \pm 122$ & -1.0 \\
\hline J11062554-7633418 & M5.5 & -0.91 & $46.05 \pm 0.15$ & $485 \pm 82$ & 5.9 & 6.4 & $21 \pm 43$ & 0.5 \\
\hline J11062942-7724586 & M6 & -1.12 & $0.25 \pm 0.16$ & $-107 \pm 79$ & -1.3 & -1.4 & $43 \pm 40$ & 1.1 \\
\hline J11063276-7625210 & M6.5 & -1.13 & $-0.01 \pm 0.16$ & $18 \pm 81$ & 0.2 & 1.0 & $81 \pm 43$ & 1.9 \\
\hline J11063945-7736052 & M5 & -0.78 & $0.37 \pm 0.16$ & $98 \pm 81$ & 1.2 & 0.7 & $34 \pm 41$ & 0.8 \\
\hline J11064180-7635489 & M5 & -0.78 & $0.97 \pm 0.16$ & $-214 \pm 84$ & -2.5 & -4.1 & $-6 \pm 43$ & -0.1 \\
\hline J11064510-7727023 & K6 & -0.03 & $0.53 \pm 0.82$ & $297 \pm 303$ & 1.0 & 0.4 & $-4 \pm 117$ & -0.0 \\
\hline J11065906-7718535 & M4.5 & -0.71 & $24.28 \pm 0.35$ & $124 \pm 77$ & 1.6 & 2.2 & $32 \pm 41$ & 0.8 \\
\hline J11065939-7530559 & M5.5 & -0.97 & $3.11 \pm 0.16$ & $-13 \pm 84$ & -0.2 & 0.4 & $138 \pm 41$ & 3.4 \\
\hline J11070925-7718471 & M3 & -0.52 & $0.06 \pm 0.82$ & $35 \pm 259$ & 0.1 & -0.2 & $92 \pm 127$ & 0.7 \\
\hline J11071181-7625501 & M5.5 & -0.97 & $0.03 \pm 0.16$ & $-50 \pm 80$ & -0.6 & -0.8 & $65 \pm 45$ & 1.4 \\
\hline J11071206-7632232 & M0 & -0.23 & $4.23 \pm 0.81$ & $195 \pm 288$ & 0.7 & 1.5 & $53 \pm 127$ & 0.4 \\
\hline J11071330-7743498 & M4 & -0.63 & $0.42 \pm 0.81$ & $-117 \pm 268$ & -0.4 & -0.6 & $18 \pm 110$ & 0.2 \\
\hline J11071860-7732516 & M5.5 & -0.92 & $0.93 \pm 0.16$ & $1 \pm 87$ & 0.0 & 0.6 & $25 \pm 43$ & 0.6 \\
\hline J11072074-7738073 & K0 & 0.29 & $26.36 \pm 1.46$ & $-597 \pm 262$ & -2.3 & -2.5 & $6 \pm 117$ & 0.1 \\
\hline J11072825-7652118 & M3 & -0.53 & $1.5 \pm 0.81$ & $-463 \pm 275$ & -1.7 & -2.4 & $10 \pm 119$ & 0.1 \\
\hline J11074245-7733593 & M5.5 & -0.88 & $2.37 \pm 0.41$ & $-110 \pm 77$ & -1.4 & -1.7 & $-58 \pm 43$ & -1.4 \\
\hline J11074366-7739411 & M1 & -0.31 & $107.27 \pm 0.56$ & $644 \pm 280$ & 2.3 & 1.7 & $110 \pm 113$ & 1.0 \\
\hline J11074656-7615174 & M6.5 & -1.15 & $2.18 \pm 0.16$ & $163 \pm 82$ & 2.0 & 2.3 & $22 \pm 45$ & 0.5 \\
\hline J11075730-7717262 & M1.2 & -0.31 & $6.47 \pm 0.8$ & $458 \pm 268$ & 1.7 & 2.0 & $-50 \pm 119$ & -0.4 \\
\hline J11075792-7738449 & K5 & -0.01 & $19.85 \pm 1.48$ & $1435 \pm 268$ & 5.6 & 3.8 & $-28 \pm 114$ & -0.3 \\
\hline J11075809-7742413 & M3 & -0.51 & $6.45 \pm 0.79$ & $-459 \pm 256$ & -1.8 & -1.1 & $-78 \pm 131$ & -0.6 \\
\hline J11080002-7717304 & K7 & -0.18 & $-0.69 \pm 0.8$ & $0 \pm 263$ & 0.0 & -0.4 & $254 \pm 109$ & 2.3 \\
\hline J11080148-7742288 & K7 & -0.2 & $44.37 \pm 0.82$ & $355 \pm 251$ & 1.4 & 1.9 & $50 \pm 122$ & 0.4 \\
\hline J11080297-7738425 & M1 & -0.2 & $102.24 \pm 0.58$ & $803 \pm 268$ & 3.0 & 4.1 & $33 \pm 115$ & 0.3 \\
\hline J11081509-7733531 & K0 & 0.12 & $209.29 \pm 0.43$ & $740 \pm 263$ & 2.8 & 2.5 & $195 \pm 119$ & 1.6 \\
\hline J11081850-7730408 & M6.5 & -1.14 & $0.26 \pm 0.16$ & $50 \pm 80$ & 0.6 & 0.5 & $-54 \pm 45$ & -1.2 \\
\hline J11082238-7730277 & M5.5 & -0.9 & $0.23 \pm 0.16$ & $78 \pm 79$ & 1.0 & 0.9 & $-2 \pm 44$ & -0.1 \\
\hline J11082570-7716396 & M8 & -1.51 & $0.23 \pm 0.15$ & $68 \pm 74$ & 0.9 & 0.6 & $-36 \pm 42$ & -0.8 \\
\hline J11082650-7715550 & M5.5 & -0.96 & $-0.24 \pm 0.16$ & $-73 \pm 79$ & -0.9 & -0.7 & $13 \pm 45$ & 0.3 \\
\hline J11083905-7716042 & K7 & -0.08 & $14.11 \pm 0.79$ & $1174 \pm 252$ & 4.7 & 6.0 & $264 \pm 111$ & 2.4 \\
\hline J11083952-7734166 & M6.5 & -0.99 & $0.02 \pm 0.16$ & $25 \pm 81$ & 0.3 & -0.3 & $38 \pm 41$ & 0.9 \\
\hline J11085090-7625135 & M5.5 & -0.9 & $-0.04 \pm 0.16$ & $-30 \pm 88$ & -0.3 & -1.0 & $-3 \pm 44$ & -0.1 \\
\hline J11085367-7521359 & M1 & -0.28 & $24.6 \pm 1.37$ & $1344 \pm 280$ & 4.8 & 5.0 & $3 \pm 122$ & 0.0 \\
\hline J11085464-7702129 & M0.5 & -0.18 & $3.9 \pm 0.79$ & $-506 \pm 255$ & -2.0 & -1.4 & $-2 \pm 127$ & -0.0 \\
\hline J11085497-7632410 & M5.5 & -0.91 & $0.46 \pm 0.16$ & $138 \pm 89$ & 1.6 & 1.8 & $100 \pm 44$ & 2.3 \\
\hline J11091812-7630292 & M0 & -0.18 & $1.3 \pm 0.79$ & $283 \pm 267$ & 1.1 & 1.7 & $-155 \pm 118$ & -1.3 \\
\hline J11092266-7634320 & M1 & -0.25 & $3.85 \pm 0.78$ & $420 \pm 274$ & 1.5 & 0.9 & $102 \pm 108$ & 0.9 \\
\hline J11092379-7623207 & M0.5 & -0.29 & $123.11 \pm 0.57$ & $192 \pm 241$ & 0.8 & 1.2 & $40 \pm 118$ & 0.3 \\
\hline J11094260-7725578 & M5 & -0.77 & $0.37 \pm 0.16$ & $38 \pm 77$ & 0.5 & 1.0 & $6 \pm 43$ & 0.1 \\
\hline J11094621-7634463 & M3 & -0.47 & $4.73 \pm 0.79$ & $213 \pm 272$ & 0.8 & 0.2 & $105 \pm 114$ & 0.9 \\
\hline J11094742-7726290 & M1 & -0.22 & $147.85 \pm 0.86$ & $293 \pm 255$ & 1.1 & 0.4 & $8 \pm 114$ & 0.1 \\
\hline J11095215-7639128 & M6.2 & -1.2 & $0.37 \pm 0.16$ & $14 \pm 76$ & 0.2 & 0.4 & $-21 \pm 39$ & -0.5 \\
\hline J11095336-7728365 & M5.5 & -0.96 & $0.29 \pm 0.16$ & $28 \pm 76$ & 0.4 & -0.9 & $24 \pm 43$ & 0.6 \\
\hline J11095340-7634255 & K7 & -0.12 & $76.1 \pm 1.83$ & $3526 \pm 270$ & 13.0 & 12.0 & $62 \pm 116$ & 0.5 \\
\hline J11095407-7629253 & M1 & -0.21 & $30.49 \pm 1.24$ & $51 \pm 279$ & 0.2 & -0.8 & $117 \pm 117$ & 1.0 \\
\hline
\end{tabular}


Table 2

(Continued)

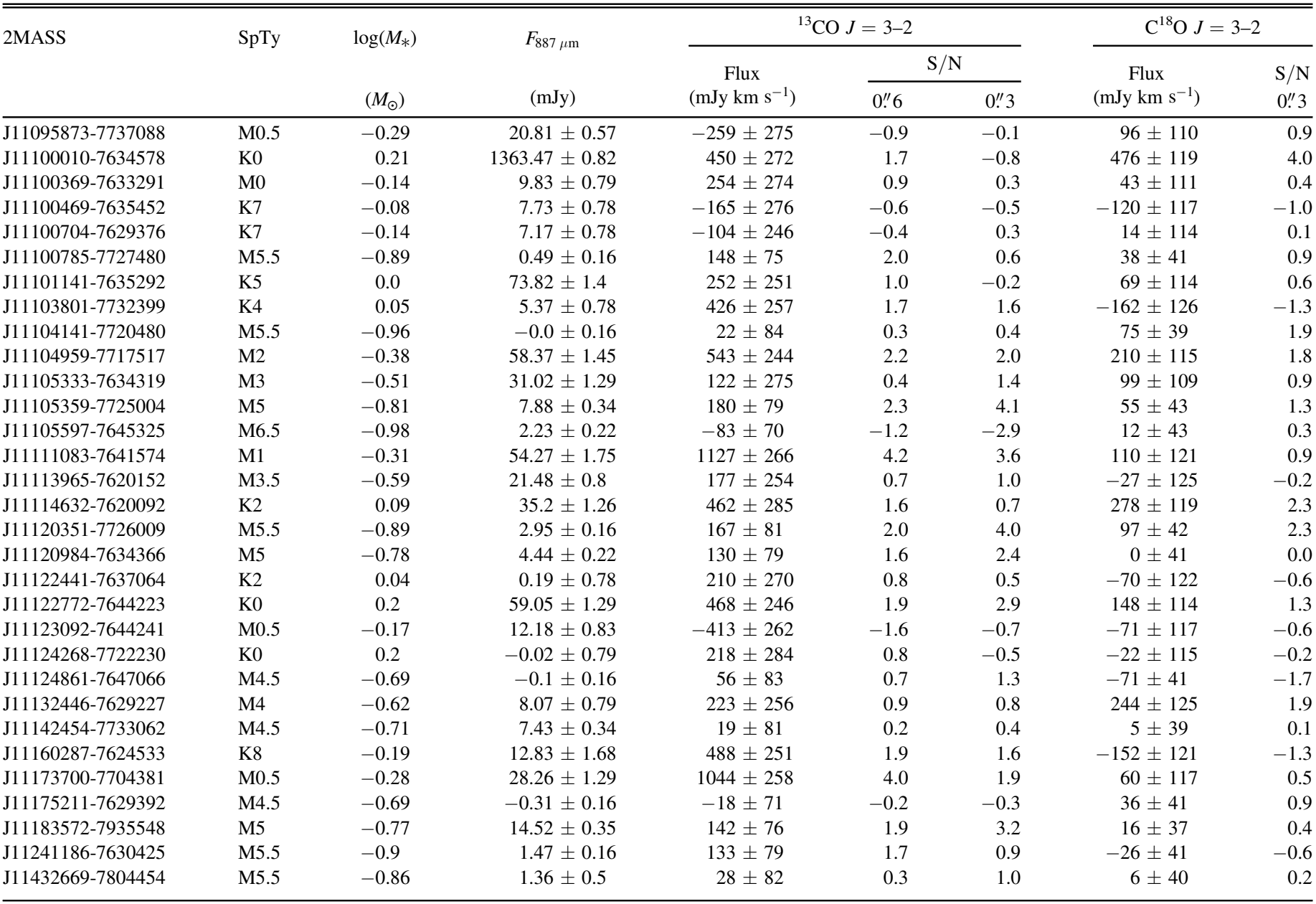

Note. The spectral type, stellar mass, and continuum flux are adopted from Pascucci et al. (2016).

location of Cha I with a distance of $206 \pm 8$ pc (excluding the

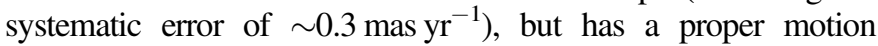
consistent with Cha II (Lopez Martí et al. 2013). The star HD 96675 is located at $161 \pm 7 \mathrm{pc}$ (again excluding systematic errors), a statistically significant outlier from the stochastic uncertainty in the Cha I distance. This Cha I distance is consistent with the $180 \pm 10$ pc measured by Voirin et al. (2017) using Gaia parallaxes combined with the distribution of reddening along the line of sight.

We adopt a distance of $157 \pm 10 \mathrm{pc}$ to Lupus, calculated from the average Gaia parallax to Lupus members with disks (Sz 68, Sz 82, RU Lup, HD 142527, RY Lup, and 2MASS J16083070-3828268). These objects have been previously identified as members of distinct regions Lupus I, Lupus II, Lupus III, and Lupus IV (Comerón 2008). The Gaia parallaxes establish that these subregions of Lupus are all located at a similar distance, in contrast to previous assumptions based on uncertain Hipparcos parallaxes. The uncertainty includes a

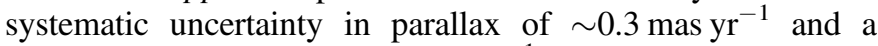
standard deviation of $0.24 \mathrm{mas} \mathrm{yr}^{-1}$ in the measurements. Several likely members ${ }^{20}$ were identified in a ROSAT X-ray

\footnotetext{
${ }^{20} \mathrm{RX}$ J1511.0-3252, HD 135127, RX J1518.4-3738, RX J1524.5-3652, RX J1529.7-3628, RX J1531.3-3329, RX J1546.6-3618, RX J1547.6-4018, RX J1549.9-3629, RX J1605.7-3905, RX J1610.0-4016, HD147402.
}

survey (Wichmann et al. 1997) and have proper motions consistent with Lupus membership (Galli et al. 2013; Gaia Collaboration et al. 2016), but have an average distance of 139 pc. Since their initial identifications and the brightnesslimited Gaia measurements may be biased to nearby objects, they are excluded from our distance analysis. These diskless members may be an older population, similar to the halo of older stars that surrounds the Taurus Molecular Cloud complexes (Kraus et al. 2017).

\section{Appendix B Maps and Comments for CO Detections \\ B.1. Comments on Individual Detections}

In this subsection, we discuss the $\mathrm{CO}$ emission properties of the two sources 2MASS J11100010-7634578 and 2MASS $\mathrm{J} 11075792-7738449$ in detail.

\section{B.1.1. 2MASS J11100010-7634578}

2MASS J11100010-7634578 displays an incomplete and asymmetric ring structure in ${ }^{13} \mathrm{CO}$ emission and is the only source detected in $\mathrm{C}^{18} \mathrm{O}$. The ${ }^{13} \mathrm{CO}$ intensity map, created by integrating the flux from 0 to $10 \mathrm{~km} \mathrm{~s}^{-1}$, shows a lack of ${ }^{13} \mathrm{CO}$ emission at the continuum center and to the NW of the disk 
Table 3

CO Fluxes and Gas Masses

\begin{tabular}{|c|c|c|c|c|c|c|c|c|c|}
\hline \multirow[t]{2}{*}{ 2MASS } & \multirow{2}{*}{$\begin{array}{c}F_{13 \mathrm{CO}} \\
\left(\mathrm{mJy} \mathrm{km} \mathrm{s}^{-1}\right)\end{array}$} & \multirow{2}{*}{$\begin{array}{l}\text { Radius } \\
\text { (arcsec) }\end{array}$} & \multirow{2}{*}{$\begin{array}{c}F_{\mathrm{C} 18 \mathrm{O}} \\
\left(\mathrm{mJy} \mathrm{km} \mathrm{s}^{-1}\right)\end{array}$} & $M_{\text {gas }}$ & $\begin{array}{c}M_{\min } \\
M v D 16\end{array}$ & $M_{\max }$ & $M_{\text {gas }}$ & $\begin{array}{l}M_{\min } \\
\text { WB14 }\end{array}$ & $M_{\max }$ \\
\hline & & & & \multicolumn{6}{|c|}{$\left(M_{\text {Jup }}\right)$} \\
\hline J10533978-7712338 & $<867$ & 0.6 & $<357$ & 0.34 & $\ldots$ & $\ldots$ & 10.48 & $\ldots$ & $\ldots$ \\
\hline J10555973-7724399 & $<885$ & 0.6 & $<351$ & 0.38 & $\ldots$ & $\ldots$ & 10.48 & $\ldots$ & $\ldots$ \\
\hline J10561638-7630530 & $<240$ & 0.6 & $<135$ & 0.08 & $\ldots$ & $\ldots$ & 1.05 & $\ldots$ & $\cdots$ \\
\hline J10563044-7711393 & $<807$ & 0.6 & $<402$ & 0.46 & $\ldots$ & $\ldots$ & 31.43 & $\ldots$ & $\ldots$ \\
\hline J10574219-7659356 & $<807$ & 0.6 & $<369$ & 0.46 & $\cdots$ & $\cdots$ & 10.48 & $\cdots$ & $\cdots$ \\
\hline J10580597-7711501 & $<234$ & 0.6 & $<129$ & 0.07 & $\ldots$ & $\ldots$ & 1.05 & $\ldots$ & $\cdots$ \\
\hline J10581677-7717170 & $2535 \pm 576$ & 1.2 & $<387$ & 0.84 & 0.19 & 5.05 & 1.57 & 1.05 & 3.14 \\
\hline J10590108-7722407 & $<867$ & 0.6 & $<375$ & 0.34 & $\ldots$ & $\ldots$ & 31.43 & $\ldots$ & $\ldots$ \\
\hline J10590699-7701404 & $1867 \pm 442$ & 0.9 & $<354$ & 0.41 & 0.13 & 4.63 & 1.3 & 1.05 & 10.48 \\
\hline J11004022-7619280 & $1004 \pm 86$ & 1.05 & $<132$ & 0.21 & 0.08 & 1.71 & 0.6 & 0.31 & 3.14 \\
\hline J11022491-7733357 & $<801$ & 0.6 & $<390$ & 0.45 & $\ldots$ & $\ldots$ & 31.43 & $\ldots$ & $\ldots$ \\
\hline J11023265-7729129 & $<840$ & 0.6 & $<387$ & 0.5 & $\ldots$ & $\ldots$ & 31.43 & $\ldots$ & $\ldots$ \\
\hline J11025504-7721508 & $<230$ & 0.6 & $<132$ & 0.07 & $\ldots$ & $\ldots$ & 1.05 & $\ldots$ & $\ldots$ \\
\hline J11040425-7639328 & $<230$ & 0.6 & $<147$ & 0.07 & $\cdots$ & $\cdots$ & 1.05 & $\cdots$ & $\cdots$ \\
\hline J11040909-7627193 & $<843$ & 0.6 & $<375$ & 0.5 & $\ldots$ & $\cdots$ & 31.43 & $\ldots$ & $\cdots$ \\
\hline J11044258-7741571 & $413 \pm 108$ & 0.75 & $<129$ & 0.08 & 0.02 & 0.19 & 0.3 & 0.1 & 3.14 \\
\hline J11045701-7715569 & $<822$ & 0.6 & $<366$ & 0.48 & $\cdots$ & $\cdots$ & 10.48 & $\cdots$ & $\cdots$ \\
\hline J11062554-7633418 & $668 \pm 143$ & 0.9 & $<129$ & 0.13 & 0.04 & 0.48 & 0.42 & 0.31 & 3.14 \\
\hline J11062942-7724586 & $<237$ & 0.6 & $<120$ & 0.07 & $\ldots$ & $\ldots$ & 1.05 & $\ldots$ & $\ldots$ \\
\hline J11063276-7625210 & $<243$ & 0.6 & $<129$ & 0.08 & $\ldots$ & $\ldots$ & 1.05 & $\ldots$ & $\ldots$ \\
\hline J11063945-7736052 & $<243$ & 0.6 & $<123$ & 0.08 & $\ldots$ & $\ldots$ & 1.05 & $\cdots$ & $\cdots$ \\
\hline J11064180-7635489 & $<252$ & 0.6 & $<129$ & 0.08 & $\ldots$ & $\ldots$ & 3.14 & $\ldots$ & $\ldots$ \\
\hline J11064510-7727023 & $<909$ & 0.6 & $<351$ & 0.45 & $\cdots$ & $\cdots$ & 10.48 & $\cdots$ & $\cdots$ \\
\hline J11065906-7718535 & $<230$ & 0.6 & $<123$ & 0.07 & $\ldots$ & $\ldots$ & 1.05 & $\ldots$ & $\ldots$ \\
\hline J11065939-7530559 & $<252$ & 0.6 & $<123$ & 0.08 & $\ldots$ & $\ldots$ & 3.14 & $\cdots$ & $\ldots$ \\
\hline J11070925-7718471 & $<777$ & 0.6 & $<381$ & 0.42 & $\cdots$ & $\cdots$ & 10.48 & $\cdots$ & $\cdots$ \\
\hline J11071181-7625501 & $<240$ & 0.6 & $<135$ & 0.08 & $\ldots$ & $\cdots$ & 1.05 & $\ldots$ & $\cdots$ \\
\hline J11071206-7632232 & $<863$ & 0.6 & $<381$ & 0.33 & $\ldots$ & $\ldots$ & 31.43 & $\ldots$ & $\ldots$ \\
\hline J11071330-7743498 & $<804$ & 0.6 & $<330$ & 0.45 & $\cdots$ & $\cdots$ & 10.48 & $\cdots$ & $\cdots$ \\
\hline J11071860-7732516 & $<261$ & 0.6 & $<129$ & 0.08 & $\ldots$ & $\cdots$ & 3.14 & $\ldots$ & $\cdots$ \\
\hline J11072074-7738073 & $<786$ & 0.6 & $<351$ & 0.43 & $\ldots$ & $\ldots$ & 10.48 & $\cdots$ & $\ldots$ \\
\hline $\mathrm{J} 11072825-7652118$ & $<825$ & 0.6 & $<357$ & 0.48 & $\ldots$ & $\ldots$ & 10.48 & $\ldots$ & $\ldots$ \\
\hline J11074245-7733593 & $<230$ & 0.6 & $<129$ & 0.07 & $\ldots$ & $\cdots$ & 1.05 & $\ldots$ & $\cdots$ \\
\hline J11074366-7739411 & $<840$ & 0.6 & $<339$ & 0.5 & $\cdots$ & $\cdots$ & 10.48 & $\cdots$ & $\cdots$ \\
\hline J11074656-7615174 & $<246$ & 0.6 & $<135$ & 0.08 & $\cdots$ & $\cdots$ & 3.14 & $\cdots$ & $\cdots$ \\
\hline J11075730-7717262 & $<804$ & 0.6 & $<357$ & 0.45 & $\cdots$ & $\cdots$ & 10.48 & $\cdots$ & $\cdots$ \\
\hline J11075792-7738449 & {$[11640 \pm 604]$} & 2.0 & $<342$ & $\ldots$ & $\ldots$ & $\ldots$ & $\ldots$ & $\cdots$ & $\cdots$ \\
\hline J11075809-7742413 & $<768$ & 0.6 & $<393$ & 0.41 & $\ldots$ & $\cdots$ & 10.48 & $\ldots$ & $\cdots$ \\
\hline J11080002-7717304 & $<789$ & 0.6 & $<327$ & 0.44 & $\ldots$ & $\cdots$ & 10.48 & $\ldots$ & $\cdots$ \\
\hline J11080148-7742288 & $<753$ & 0.6 & $<366$ & 0.4 & $\ldots$ & $\ldots$ & 10.48 & $\ldots$ & $\ldots$ \\
\hline J11080297-7738425 & $624 \pm 188$ & 0.45 & $<345$ & 0.12 & 0.03 & 0.48 & 0.59 & 0.1 & 10.48 \\
\hline J11081509-7733531 & $978 \pm 326$ & 0.75 & $<357$ & 0.2 & 0.05 & 4.17 & 0.91 & 0.31 & 10.48 \\
\hline J11081850-7730408 & $<240$ & 0.6 & $<135$ & 0.08 & $\ldots$ & $\ldots$ & 1.05 & $\ldots$ & $\ldots$ \\
\hline J11082238-7730277 & $<237$ & 0.6 & $<132$ & 0.07 & $\ldots$ & $\ldots$ & 1.05 & $\ldots$ & $\ldots$ \\
\hline J11082570-7716396 & $<221$ & 0.6 & $<126$ & 0.07 & $\ldots$ & $\cdots$ & 1.05 & $\ldots$ & $\cdots$ \\
\hline J11082650-7715550 & $<237$ & 0.6 & $<135$ & 0.07 & $\cdots$ & $\cdots$ & 1.05 & $\cdots$ & $\ldots$ \\
\hline J11083905-7716042 & $1252 \pm 342$ & 0.75 & $<333$ & 0.26 & 0.08 & 4.36 & 1.09 & 0.31 & 10.48 \\
\hline J11083952-7734166 & $<243$ & 0.6 & $<123$ & 0.08 & $\ldots$ & $\ldots$ & 1.05 & $\ldots$ & $\ldots$ \\
\hline J11085090-7625135 & $<264$ & 0.6 & $<132$ & 0.08 & $\ldots$ & $\ldots$ & 3.14 & $\ldots$ & $\cdots$ \\
\hline J11085367-7521359 & $2634 \pm 527$ & 1.05 & $<366$ & 0.89 & 0.21 & 4.78 & 1.53 & 1.05 & 3.14 \\
\hline J11085464-7702129 & $<765$ & 0.6 & $<381$ & 0.41 & $\ldots$ & $\ldots$ & 10.48 & $\ldots$ & $\ldots$ \\
\hline J11085497-7632410 & $<267$ & 0.6 & $<132$ & 0.08 & $\ldots$ & $\ldots$ & 3.14 & $\cdots$ & $\cdots$ \\
\hline J11091812-7630292 & $<801$ & 0.6 & $<354$ & 0.45 & $\ldots$ & $\ldots$ & 10.48 & $\ldots$ & $\ldots$ \\
\hline J11092266-7634320 & $<822$ & 0.6 & $<324$ & 0.48 & $\cdots$ & $\cdots$ & 10.48 & $\cdots$ & $\cdots$ \\
\hline J11092379-7623207 & $<723$ & 0.6 & $<354$ & 0.37 & $\ldots$ & $\ldots$ & 10.48 & $\cdots$ & $\cdots$ \\
\hline J11094260-7725578 & $<230$ & 0.6 & $<129$ & 0.07 & $\ldots$ & $\ldots$ & 1.05 & $\ldots$ & $\ldots$ \\
\hline J11094621-7634463 & $<816$ & 0.6 & $<342$ & 0.47 & $\cdots$ & $\cdots$ & 10.48 & $\cdots$ & $\cdots$ \\
\hline J11094742-7726290 & $<765$ & 0.6 & $<342$ & 0.41 & $\ldots$ & $\ldots$ & 10.48 & $\ldots$ & $\ldots$ \\
\hline J11095215-7639128 & $<227$ & 0.6 & $<117$ & 0.07 & $\cdots$ & $\cdots$ & 1.05 & $\cdots$ & $\cdots$ \\
\hline J11095336-7728365 & $<227$ & 0.6 & $<129$ & 0.07 & $\cdots$ & $\ldots$ & 1.05 & $\ldots$ & $\ldots$ \\
\hline J11095340-7634255 & $5373 \pm 450$ & 0.9 & $<348$ & 4.55 & 0.36 & 104.76 & 15.98 & 3.14 & 104.76 \\
\hline J11095407-7629253 & $<837$ & 0.6 & $<351$ & 0.49 & $\ldots$ & $\ldots$ & 10.48 & $\ldots$ & $\ldots$ \\
\hline
\end{tabular}


Table 3

(Continued)

\begin{tabular}{|c|c|c|c|c|c|c|c|c|c|}
\hline \multirow[t]{2}{*}{ 2MASS } & \multirow{2}{*}{$\begin{array}{c}F_{13 \mathrm{CO}} \\
\left(\mathrm{mJy} \mathrm{km} \mathrm{s}^{-1}\right)\end{array}$} & \multirow{2}{*}{$\begin{array}{l}\text { Radius } \\
\text { (arcsec) }\end{array}$} & \multirow{2}{*}{$\begin{array}{c}F_{\mathrm{C} 18 \mathrm{O}} \\
\left(\mathrm{mJy} \mathrm{km} \mathrm{s}^{-1}\right)\end{array}$} & $M_{\text {gas }}$ & $\begin{array}{c}M_{\min } \\
\text { MvD16 }\end{array}$ & $M_{\max }$ & $M_{\text {gas }}$ & $\begin{array}{l}M_{\min } \\
\text { WB14 }\end{array}$ & $M_{\max }$ \\
\hline & & & & \multicolumn{6}{|c|}{$\left(M_{\mathrm{Jup}}\right)$} \\
\hline J11095873-7737088 & $<825$ & 0.6 & $<330$ & 0.48 & $\ldots$ & $\cdots$ & 10.48 & $\cdots$ & $\cdots$ \\
\hline J11100010-7634578 & $1614 \pm 503$ & 1.05 & $1581 \pm 343$ & 18.01 & 2.14 & 76.22 & 51.43 & 3.14 & 104.76 \\
\hline J11100369-7633291 & $<822$ & 0.6 & $<333$ & 0.48 & $\cdots$ & $\ldots$ & 10.48 & $\cdots$ & $\ldots$ \\
\hline J11100469-7635452 & $<828$ & 0.6 & $<351$ & 0.48 & $\ldots$ & $\ldots$ & 10.48 & $\ldots$ & $\ldots$ \\
\hline J11100704-7629376 & $<738$ & 0.6 & $<342$ & 0.38 & $\cdots$ & $\cdots$ & 10.48 & $\cdots$ & $\cdots$ \\
\hline J11100785-7727480 & $<224$ & 0.6 & $<123$ & 0.07 & $\cdots$ & $\cdots$ & 1.05 & $\cdots$ & $\cdots$ \\
\hline J11101141-7635292 & $<753$ & 0.6 & $<342$ & 0.4 & $\ldots$ & $\ldots$ & 10.48 & $\cdots$ & $\cdots$ \\
\hline J11103801-7732399 & $<771$ & 0.6 & $<378$ & 0.42 & $\cdots$ & $\cdots$ & 10.48 & $\cdots$ & $\cdots$ \\
\hline J11104141-7720480 & $<252$ & 0.6 & $<117$ & 0.08 & $\cdots$ & $\cdots$ & 3.14 & $\cdots$ & $\cdots$ \\
\hline J11104959-7717517 & $<732$ & 0.6 & $<345$ & 0.37 & $\ldots$ & $\ldots$ & 10.48 & $\ldots$ & $\ldots$ \\
\hline J11105333-7634319 & $<825$ & 0.6 & $<327$ & 0.48 & $\ldots$ & $\ldots$ & 10.48 & $\cdots$ & $\cdots$ \\
\hline J11105359-7725004 & $173 \pm 54$ & 0.45 & $<129$ & 0.03 & 0.01 & 0.07 & 0.14 & 0.1 & 1.05 \\
\hline J11105597-7645325 & $<210$ & 0.6 & $<129$ & 0.07 & $\cdots$ & $\cdots$ & 1.05 & $\cdots$ & $\cdots$ \\
\hline J11111083-7641574 & $1913 \pm 440$ & 0.9 & $<363$ & 0.42 & 0.14 & 4.75 & 1.31 & 1.05 & 10.48 \\
\hline J11113965-7620152 & $<762$ & 0.6 & $<375$ & 0.41 & $\cdots$ & $\cdots$ & 10.48 & $\cdots$ & $\ldots$ \\
\hline J11114632-7620092 & $<855$ & 0.6 & $<357$ & 0.51 & $\cdots$ & $\cdots$ & 10.48 & $\cdots$ & $\cdots$ \\
\hline J11120351-7726009 & $165 \pm 54$ & 0.45 & $<126$ & 0.03 & 0.01 & 0.07 & 0.13 & 0.1 & 1.05 \\
\hline J11120984-7634366 & $<237$ & 0.6 & $<123$ & 0.07 & $\cdots$ & $\cdots$ & 1.05 & $\cdots$ & $\cdots$ \\
\hline J11122441-7637064 & $<810$ & 0.6 & $<366$ & 0.46 & $\cdots$ & $\cdots$ & 10.48 & $\cdots$ & $\cdots$ \\
\hline J11122772-7644223 & $<738$ & 0.6 & $<342$ & 0.38 & $\cdots$ & $\cdots$ & 10.48 & $\cdots$ & $\cdots$ \\
\hline J11123092-7644241 & $<786$ & 0.6 & $<351$ & 0.43 & $\cdots$ & $\cdots$ & 10.48 & $\cdots$ & $\cdots$ \\
\hline J11124268-7722230 & $<851$ & 0.6 & $<345$ & 0.51 & $\ldots$ & $\ldots$ & 10.48 & $\ldots$ & $\ldots$ \\
\hline J11124861-7647066 & $<249$ & 0.6 & $<123$ & 0.08 & $\ldots$ & $\ldots$ & 3.14 & $\ldots$ & $\ldots$ \\
\hline J11132446-7629227 & $<768$ & 0.6 & $<375$ & 0.41 & $\cdots$ & $\cdots$ & 10.48 & $\cdots$ & $\cdots$ \\
\hline J11142454-7733062 & $<243$ & 0.6 & $<117$ & 0.08 & $\cdots$ & $\cdots$ & 1.05 & $\cdots$ & $\cdots$ \\
\hline J11160287-7624533 & $<753$ & 0.6 & $<363$ & 0.4 & $\cdots$ & $\cdots$ & 10.48 & $\cdots$ & $\cdots$ \\
\hline J11173700-7704381 & $2170 \pm 476$ & 1.05 & $<351$ & 0.48 & 0.16 & 4.59 & 1.35 & 1.05 & 3.14 \\
\hline J11175211-7629392 & $<212$ & 0.6 & $<123$ & 0.07 & $\cdots$ & $\cdots$ & 1.05 & $\cdots$ & $\cdots$ \\
\hline J11183572-7935548 & $164 \pm 50$ & 0.45 & $<110$ & 0.03 & 0.01 & 0.07 & 0.13 & 0.1 & 1.05 \\
\hline J11241186-7630425 & $<237$ & 0.6 & $<123$ & 0.07 & $\cdots$ & $\cdots$ & 1.05 & $\cdots$ & $\cdots$ \\
\hline J11432669-7804454 & $<246$ & 0.6 & $<120$ & 0.08 & $\cdots$ & $\cdots$ & 3.14 & $\cdots$ & $\cdots$ \\
\hline
\end{tabular}

Note. The aperture radii for ${ }^{13} \mathrm{CO}$ flux calculations are listed in the third column. Upper limits for $\mathrm{C}^{18} \mathrm{O}$ fluxes are calculated from the $0 .{ }^{\prime \prime} 3$ radius apertures, with the only exception, 2MASS J11100010-7634578, adopted with a $0 . " 75$ radius aperture.

(Figure 11, upper left). To understand this peculiar distribution of ${ }^{13} \mathrm{CO}$ emission, we extract four spectra from a 0.15 radius aperture in the marked positions of the intensity map. Positions a and c correspond to the red-and blueshifted components to the NE and SW directions, respectively, indicating a Keplerianlike disk structure (see also the velocity map in Figure 11). The flux difference in SW and NE directions is attributed to the apparent negative flux around $5 \mathrm{~km} \mathrm{~s}^{-1}$ in the blueshifted component (marker c). The reason for negative flux in spectra $\mathrm{c}$ and $\mathrm{d}$ is unclear and may be caused by either random noise fluctuations or perhaps foreground absorption in Cha I clouds. The $\mathrm{C}^{18} \mathrm{O}$ emission is more compact toward the source center than ${ }^{13} \mathrm{CO}$ and is not affected by the negative fluxes.

The flux ratio of ${ }^{13} \mathrm{CO}$ to $\mathrm{C}^{18} \mathrm{O}$ emission is $\sim 1$, when extracting fluxes over the $0-10 \mathrm{~km} \mathrm{~s}^{-1}$ spectral range and the same aperture of $1.05 \mathrm{arcsec}$. If the velocity range from 4 to $6 \mathrm{~km} \mathrm{~s}^{-1}$ is excluded from both spectra, the ${ }^{13} \mathrm{CO}-$ to- $\mathrm{C}^{18} \mathrm{O}$ flux ratio increases to $\sim 2$. If the ${ }^{13} \mathrm{CO}$ emission is optically thick where the submillimeter continuum is produced, then the continuum emission under the line would be absorbed and lower the line flux after continuum subtraction (see supplementary material in Isella et al. 2016). At any given location in the disk, the line would be most opaque within the turbulent velocity of $\sim 0.5 \mathrm{~km} \mathrm{~s}^{-1}$ (e.g., Hughes et al. 2011). Correcting the ${ }^{13} \mathrm{CO}$ flux for this effect would increase the line flux by $\sim 30 \%$.

2MASS J11100010-7634578 is thought to drive the highly collimated jet $\mathrm{HH} 915$, with a P.A. $\sim 135^{\circ}$ (Schegerer et al. 2006). Two near-infrared $\mathrm{H}_{2}$ emission knots (marked as $\mathrm{A}$ and $\mathrm{D}$ in the velocity map in Figure 11 ), located to the NW of the source location, were suggested as counterparts of the HH 915 object (Wang \& Henning 2006). The jet direction is perpendicular to the red- and blueshifted component direction (in NE-SW), as depicted in the velocity map. Therefore, the asymmetric ${ }^{13} \mathrm{CO}$ emission likely traces the Keplerian rotation of disk materials, with the NW part of the disk contaminated by high noise fluctuation or strong absorption.

This source has a stellar companion discovered from near-IR interferometry with a projected separation of $1 \mathrm{au}$ (Anthonioz et al. 2015), which is too close to affect the disk at the large spatial scales detectable in our ALMA observations. However, the presence of a companion may affect the disk dispersal timescale and therefore the disk mass and size (e.g., Harris et al. 2012; Kraus et al. 2012).

\section{B.1.2. 2MASS J11075792-7738449}

While submillimeter continuum emission is clearly detected at the source position of 2MASS J11075792-7738449, the 

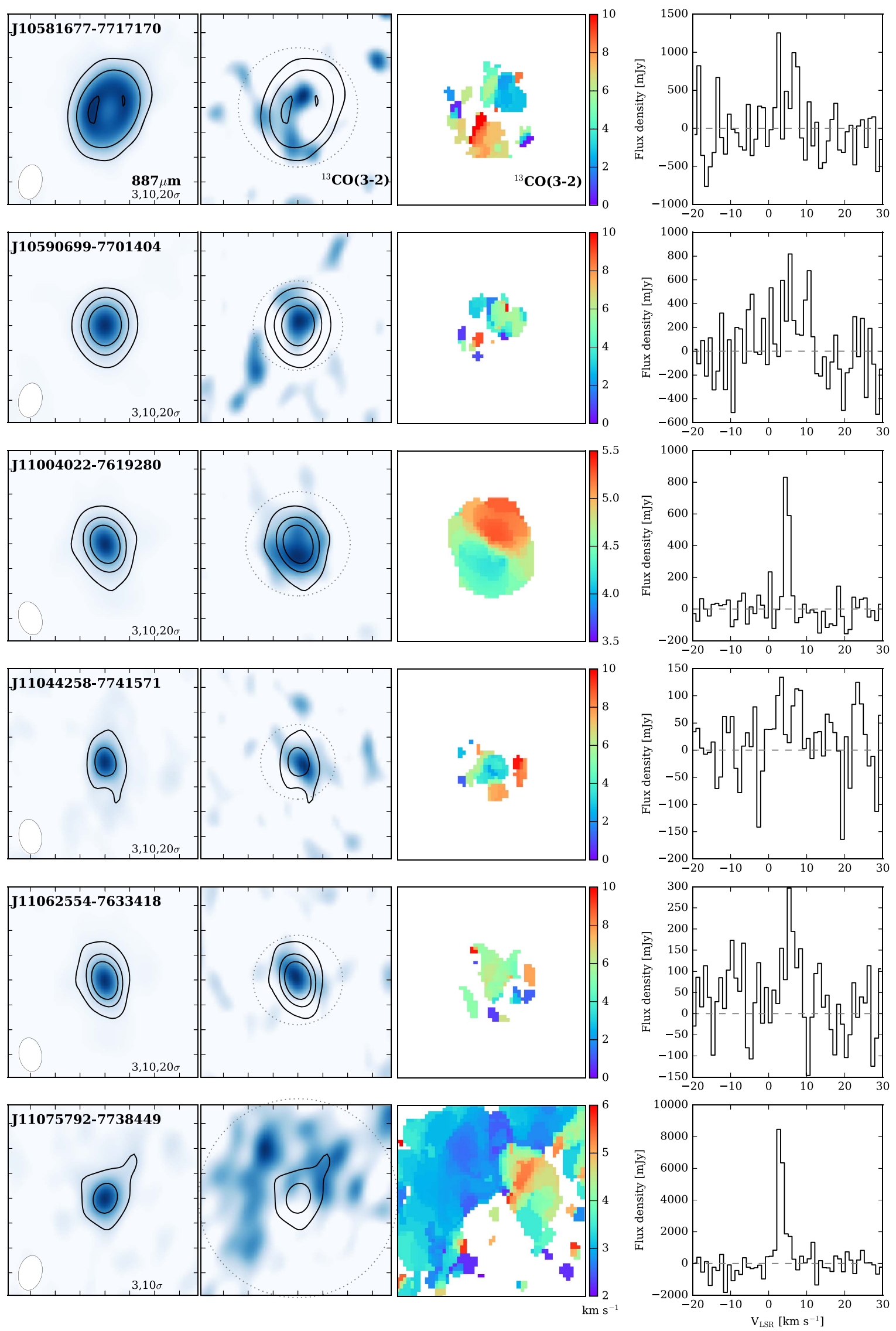

Figure 10. From left to right: ALMA $887 \mu$ m continuum image, ${ }^{13} \mathrm{CO}$ moment 0 map, ${ }^{13} \mathrm{CO}$ moment 1 map with $3 \sigma$ cutoff, and ${ }^{13} \mathrm{CO}$ spectrum with extraction region shown as a dashed circle in the moment 0 map for ${ }^{13} \mathrm{CO}$ detections in Cha I observed by our ALMA Cycle 2 program. Images are $4^{\prime \prime} \times 4^{\prime \prime}$ in size. Synthesized beams are shown in the lower right corner of the continuum images. 

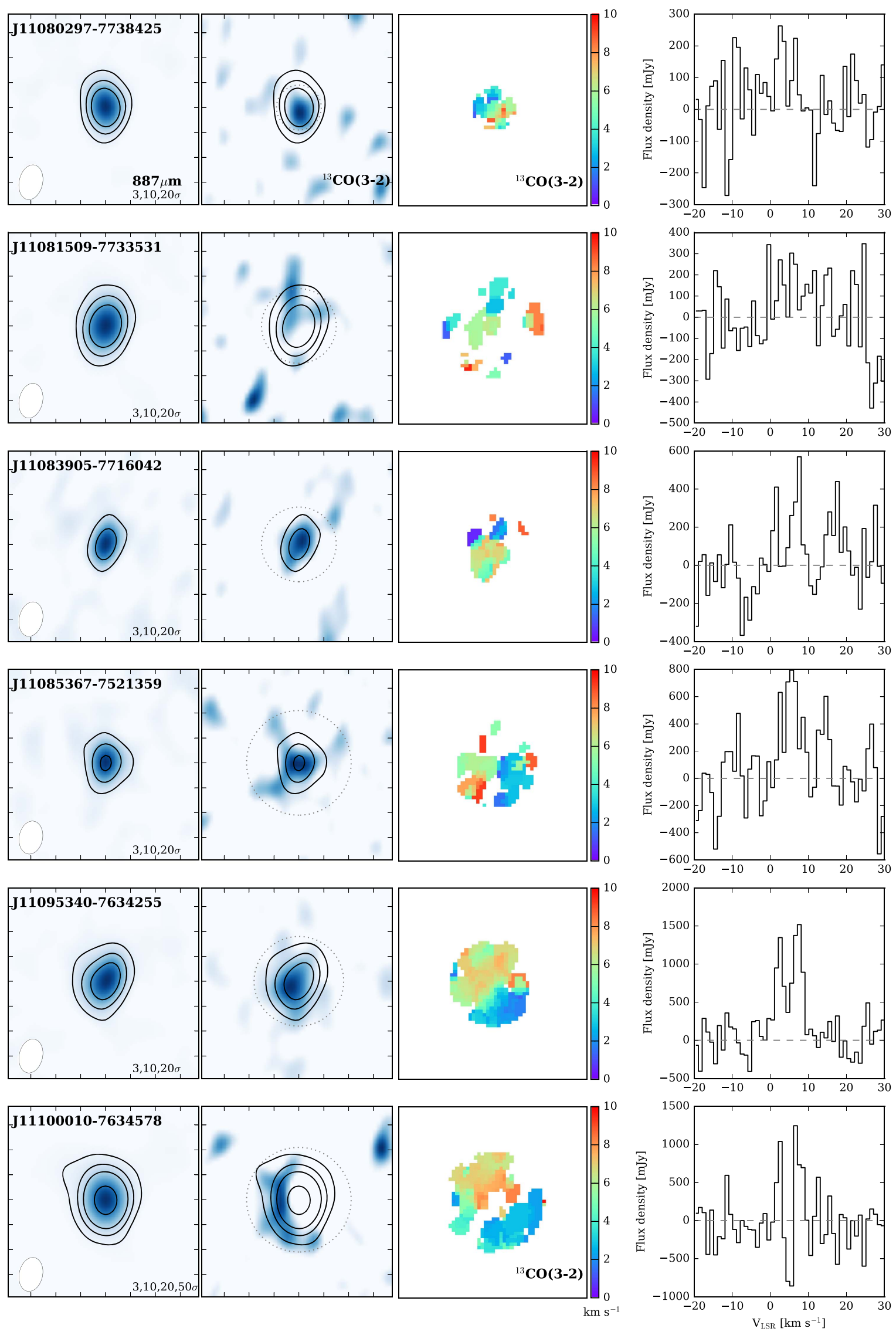

Figure 10. (Continued.) 

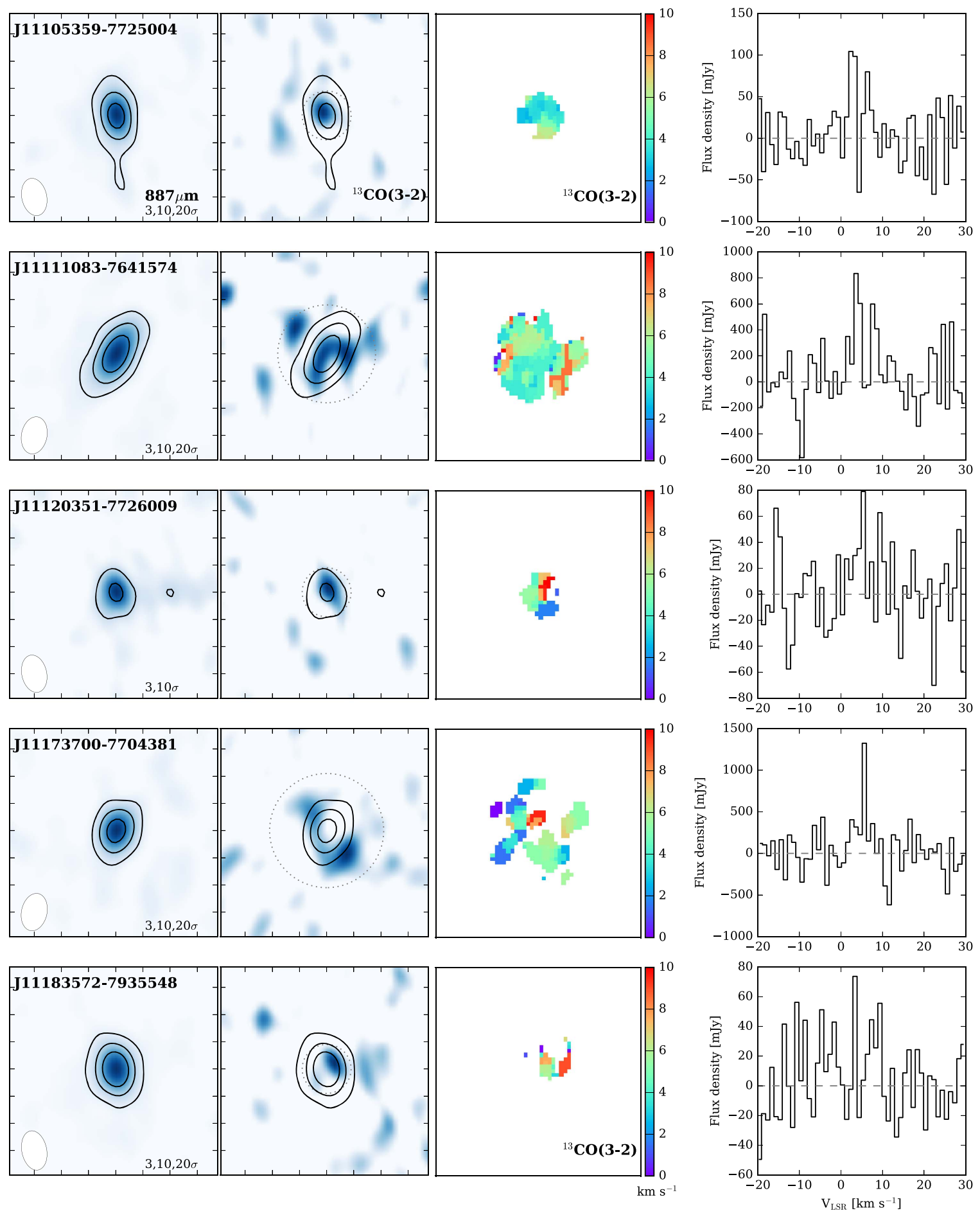

Figure 10. (Continued.)

associated ${ }^{13} \mathrm{CO}$ emission is completely offset from its continuum center, as shown in Figure 12 (see also the channel map in Figure 13). The ${ }^{13} \mathrm{CO}$ emission peaks to the $\mathrm{NE}$ of the source position by $\sim 1^{\prime \prime}$ (component a) and extends to the south by $3^{\prime \prime}$ (component b).

High-resolution optical imaging with HST reveals a reflection nebulosity associated with the object (Schmidt et al. 2013), shown as the background in the right panel of Figure 12. Schmidt et al. (2013) argues that the reflection nebulosity is illuminated by the Herbig A0 star HD 97048, separated by $37^{\prime \prime}(0.034 \mathrm{pc}$ for a
Cha I distance of $188 \mathrm{pc}$ ) to the SE. The extended ${ }^{13} \mathrm{CO}$ emission (components $\mathrm{a}$ and $\mathrm{b}$ ) is spatially consistent with the reflection nebulosity. The measured ${ }^{13} \mathrm{CO}$ flux increases significantly when including visibilities with baselines $<40 \mathrm{k} \lambda$. Since short baselines trace extended emission, the increased flux may originate from the nebula or nearby clouds. This origin may also be the most likely cause for component $\mathrm{d}$, since no optical or submillimeter source is associated with it.

Schmidt et al. (2013) discovered an $\sim \mathrm{M} 4.5$ companion, which is located $0 \prime \prime 5(\sim 94 \mathrm{au}) \mathrm{W}$ of the primary star and has a 

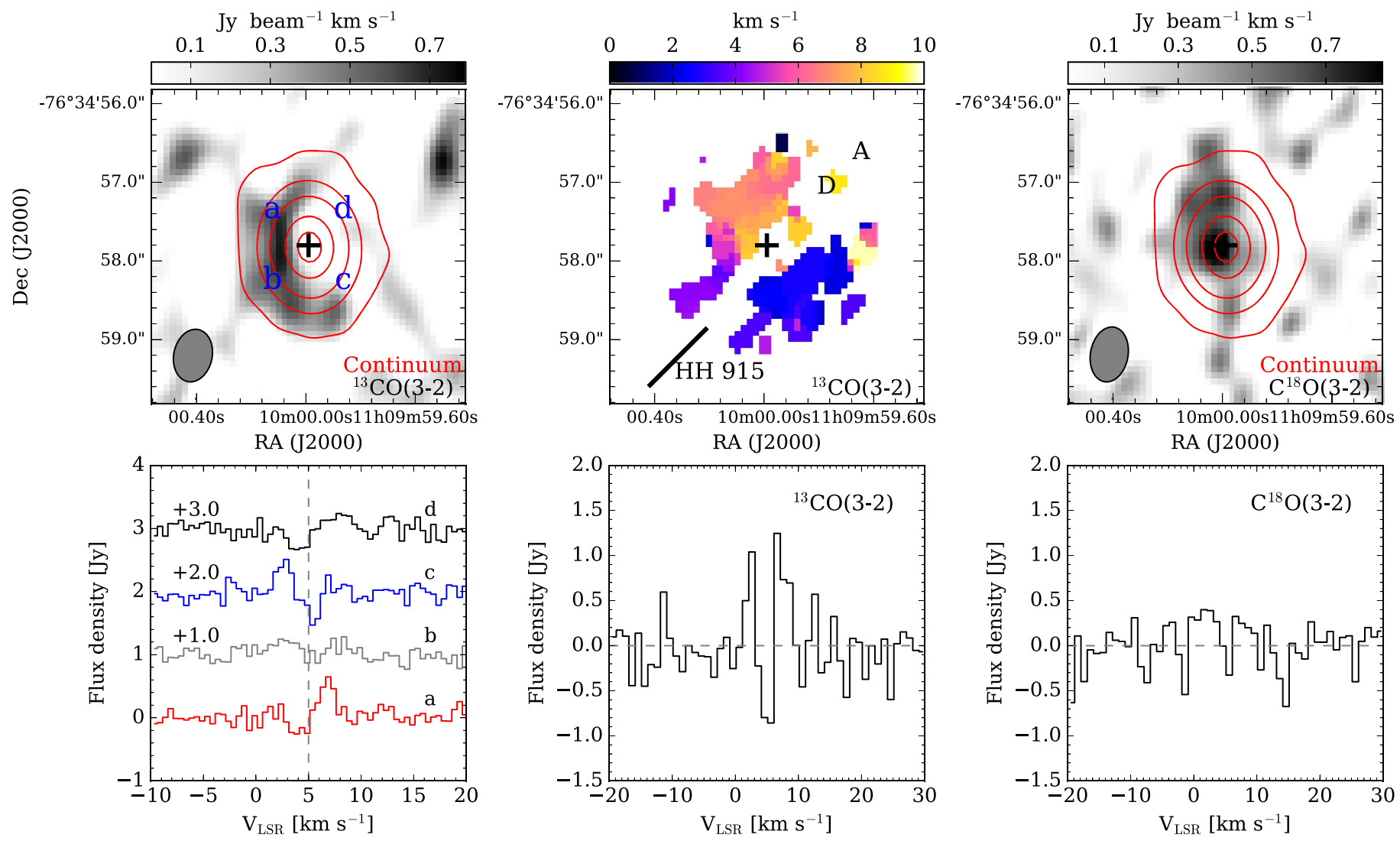

Figure 11. 2MASS J11100010-7634578: integrated intensity maps (moment 0 map) of ${ }^{13} \mathrm{CO}$ and $\mathrm{C}^{18} \mathrm{O}$ are shown in the top left and top right panels, respectively, with $887 \mu$ m continuum contours at $[3,30,100,300,500] \sigma$, with $1 \sigma \sim 1$ mJy beam $^{-1}$. The velocity map (moment 1 map) of ${ }^{13} \mathrm{CO}$ is in the top middle panel. The $\mathrm{HH}$ object position associated with this source is noted in the velocity map, as well as the two $\mathrm{H}_{2}$ emission knots, A and D. Four spectra are extracted from the a, b, c, and $\mathrm{d}$ positions with a $0 . " 5$ radius aperture from the ${ }^{13} \mathrm{CO}$ intensity map. Line spectra for ${ }^{13} \mathrm{CO}$ and $\mathrm{C}^{18} \mathrm{O}$ are also shown as a comparison, extracted from determined aperture sizes of 1.05 and 0.75 arcsec, respectively.
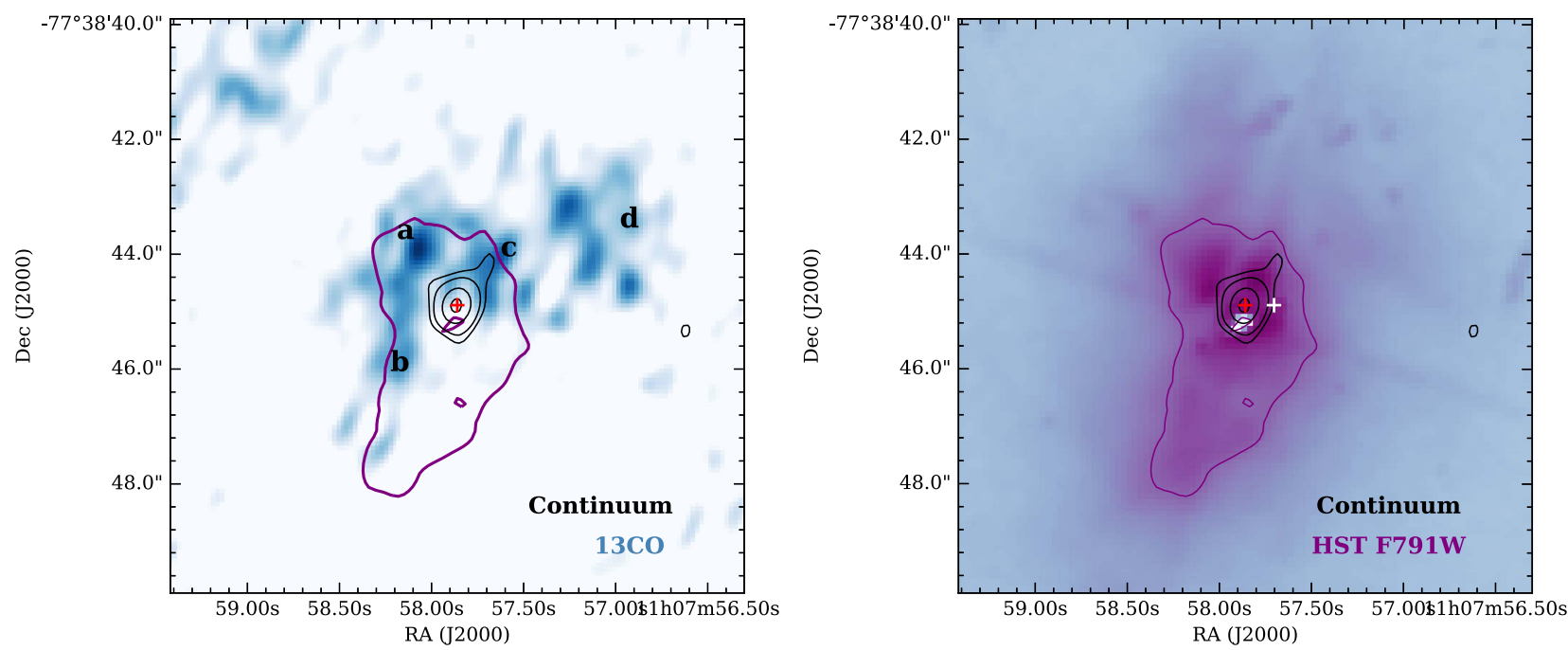

Figure 12. 2MASS J11075792-7738449. Left: ${ }^{13} \mathrm{CO}$ intensity map, integrated from 2 to $6 \mathrm{~km} \mathrm{~s}^{-1}$. Continuum emission is also shown in black contours, with contour levels of $[3,5,10,15] \sigma$. The source position and the newly found 0.5 arcsec companion are marked with red and white plus signs, respectively. Four emission components are labeled as a, b, c, and d. Right: circumstellar disk/reflection nebula in HST filter F791W. The purple contour depicts the nebula edge at 3\% peak level.

proper motion suggesting either an edge-on or a highly eccentric orbit. Since the dust disk has survived, the lack of ${ }^{13} \mathrm{CO}$ emission in the dust disk therefore should not be related to any disk dispersal caused by the companion. The detected
${ }^{13} \mathrm{CO}$ emission may not trace the disk, but the nearby clouds instead. More sensitive observations are needed to understand the ${ }^{13} \mathrm{CO}$ emission from and around 2MASS J110757927738449 . 


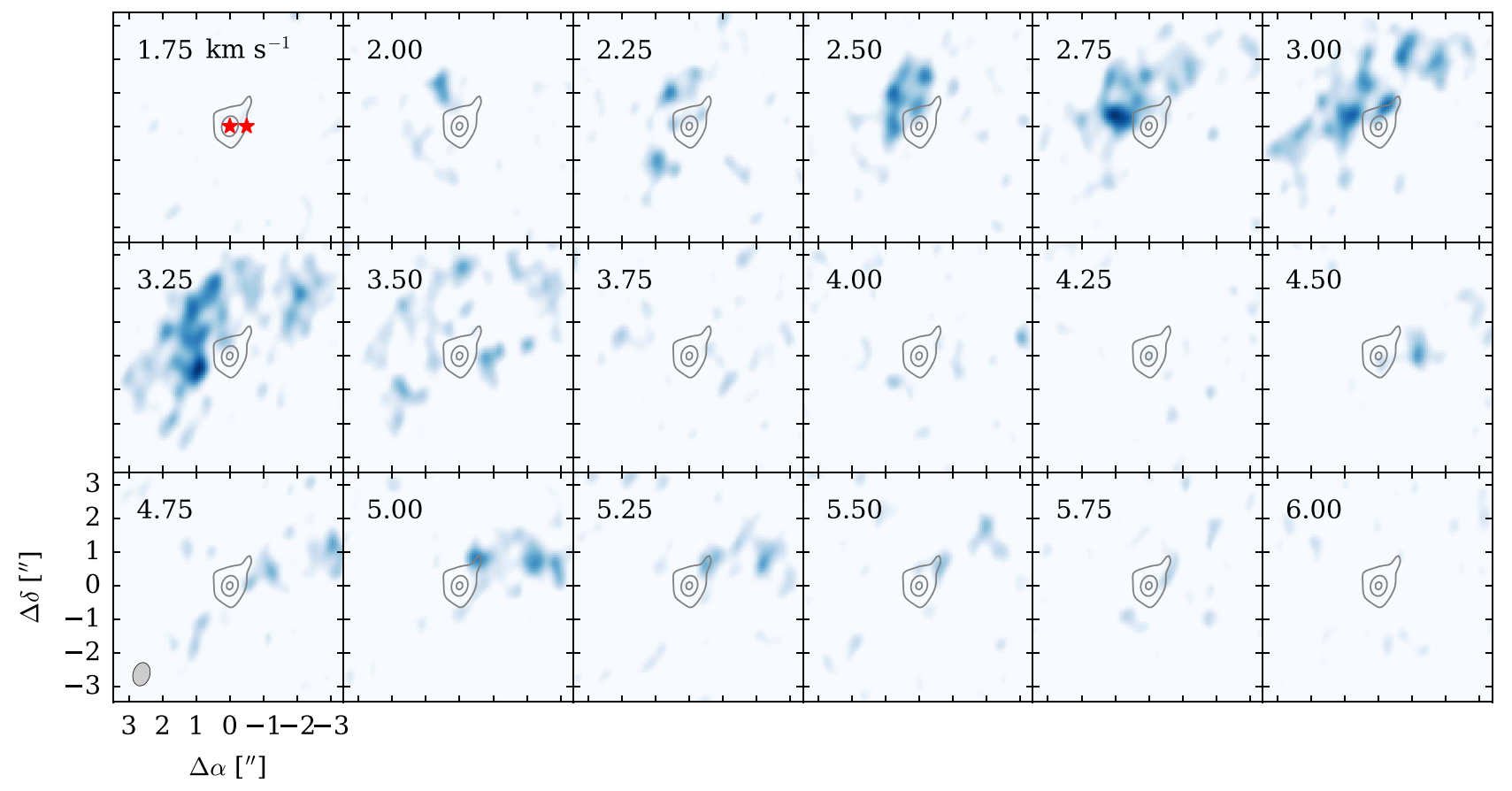

Figure 13. 2MASS J11075792-7738449 channel map with channel velocity shown in the upper left corner. Continuum emission is shown in black contours, with contour levels of $[3,10,15] \sigma$. The source position and the newly found 0.5 arcsec companion are marked with red stars.

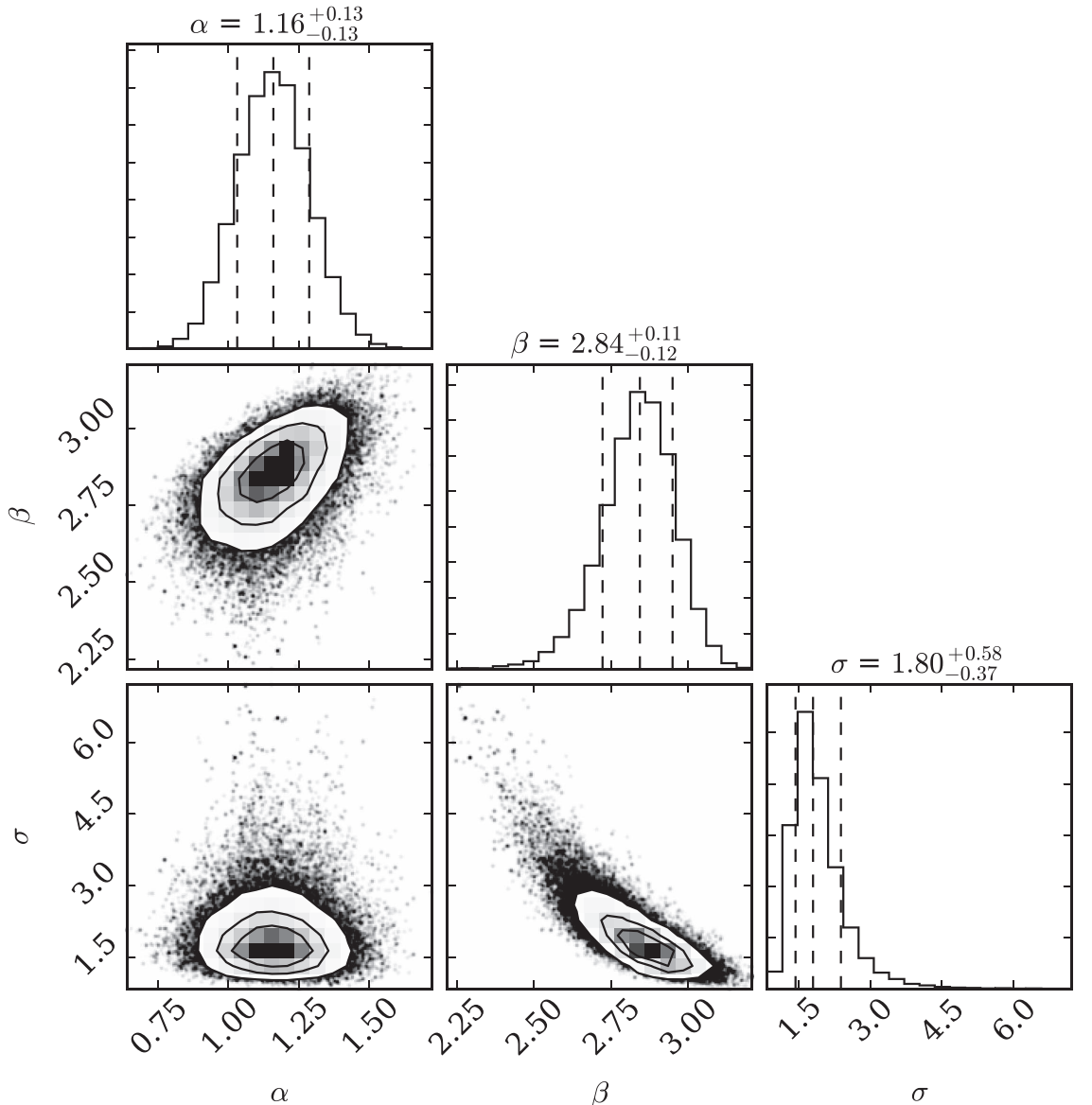

Figure 14. Corner plot of the $\log F_{13 \mathrm{CO}}$ vs. $\log M_{*}$ fit with model $F_{13 \mathrm{CO}}=\beta \cdot M_{*}^{\alpha}$. 

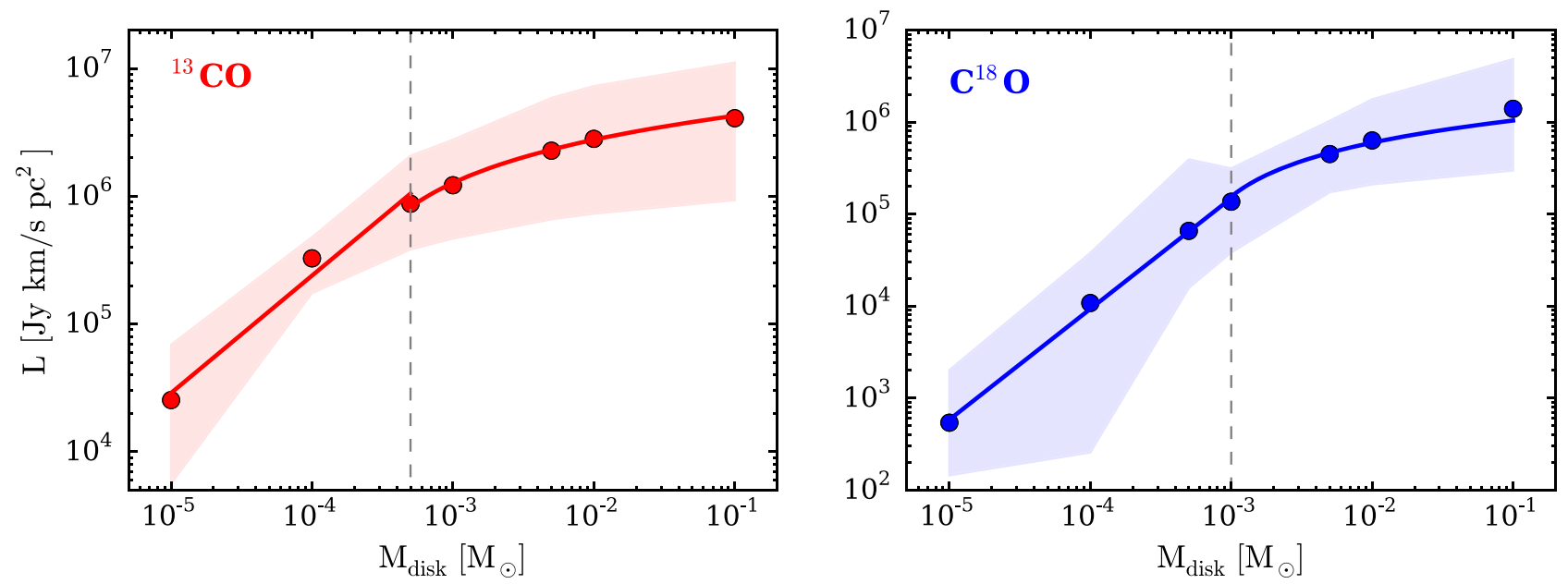

Figure 15. Fitting the median line luminosity in each disk mass bin with disk gas mass for ${ }^{13} \mathrm{CO}$ (left) and $\mathrm{C}^{18} \mathrm{O}$ (right). The transition masses for ${ }^{13} \mathrm{CO}$ and $\mathrm{C}^{18} \mathrm{O}$ fitting functions are shown as gray dashed lines. The data used here were obtained from Miotello et al. (2016).

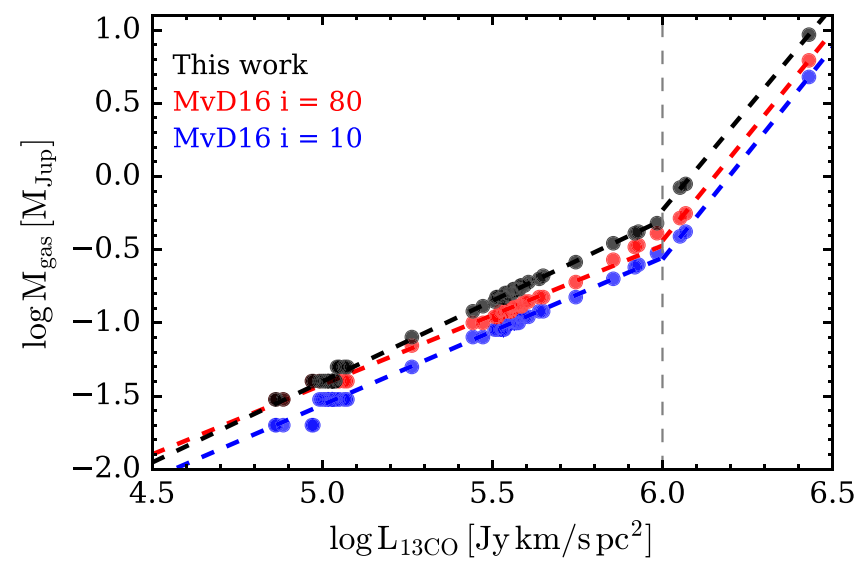

Figure 16. Comparison of gas masses for our Cha I sample, derived using different fitting functions. The scaling relation breaks at the ${ }^{13} \mathrm{CO}$ luminosity $\sim 10^{6} L_{\odot}$.

Table 4

Coefficients in Line Luminosity and Gas Mass Fitting

\begin{tabular}{cccccc}
\hline \hline & $A$ & $B$ & $C$ & $D$ & $M_{\mathrm{tr}}\left(M_{\odot}\right)$ \\
\hline${ }^{13} \mathrm{CO}$ & 9.054 & 0.919 & $5.765 \cdot 10^{6}$ & $1.498 \cdot 10^{6}$ & $5 \cdot 10^{-4}$ \\
$\mathrm{C}^{18} \mathrm{O}$ & 8.783 & 1.203 & $1.484 \cdot 10^{6}$ & $4.428 \cdot 10^{5}$ & $1 \cdot 10^{-3}$ \\
\hline
\end{tabular}

Note. The fitting functions between ${ }^{13} \mathrm{CO}$ and $\mathrm{C}^{18} \mathrm{O}$ line luminosity and disk gas mass using model data in $\operatorname{MvD} 16: \log \left(L_{\mathrm{CO}}\right)=A+B \cdot \log \left(M_{\text {gas }}\right)$ when $M_{\text {gas }}$ $\leqslant M_{\mathrm{tr}} ; L_{\mathrm{CO}}=C+D \cdot \log \left(M_{\mathrm{gas}}\right)$ when $M_{\mathrm{gas}} \geqslant M_{\mathrm{tr}}$.

\section{Appendix C \\ Corner Plots of the Fit}

We show in Figure 14 the corner plots with slope, intercept, and intrinsic scatter for the fit of the $\log F_{13 \mathrm{CO}}$ versus $\log M_{*}$ relation (see Section 4.2 and Figure 6). This is done using model $F_{13 \mathrm{CO}}=\beta \cdot M_{*}^{\alpha}$, with nondetections applying calculated fluxes instead of $3 \sigma$ upper limits.

\section{Appendix D \\ Line Luminosity versus Disk Mass}

With the model grids from MvD16, we fit simple functions for line luminosities and disk gas masses using the median ${ }^{13} \mathrm{CO}$ and $\mathrm{C}^{18} \mathrm{O} J=3-2$ line luminosities in each gas mass bin. Similar to the methods of Miotello et al. (2016, 2017), we fit a linear relation for line luminosity and gas mass in the low-mass regime and a logarithmic relation in the higher-mass end. Compared to the recent analysis of Miotello et al. (2017), published during the final phases of preparation of this paper, we fit a linear relation in the $\log -\log$ plane and choose a different transition mass. The fitting coefficients are listed in Table 4 . We calculate gas masses for our ${ }^{13} \mathrm{CO}$ detections using our fitted coefficients and the values provided in Miotello et al. (2017), in which models with disk inclinations of $10^{\circ}$ and $80^{\circ}$ are fitted separately. As shown in Figure 16, the derived gas masses are almost consistent. In most cases, the gas mass derived using our fitted functions should be reduced by factors of 1.6 (for models with inclination angle $10^{\circ}$ ) and 1.2 (for $80^{\circ}$ ) for consistency with the results in Miotello et al. (2017). The discrepancies between the two methods are slightly larger for the few brightest sources in our sample.

\section{References}

Aikawa, Y., van Zadelhoff, G. J., van Dishoeck, E. F., \& Herbst, E. 2002, A\&A, 386, 622

Akritas, M. G., \& Bershady, M. A. 1996, ApJ, 470, 706

Alexander, R., Pascucci, I., Andrews, S., Armitage, P., \& Cieza, L. 2014, in Protostars and Planets VI, ed. H. Beuther, R. S. Klessen, C. P. Dullemond, \& T. Henning (Tucson, AZ: Univ. of Arizona Press), 475

Andrews, S. M., Rosenfeld, K. A., Kraus, A. L., \& Wilner, D. J. 2013, ApJ, 771,129

Andrews, S. M., Wilner, D. J., Hughes, A. M., et al. 2012, ApJ, 744, 162

Ansdell, M., Williams, J. P., Manara, C. F., et al. 2017, arXiv:1703.08546

Ansdell, M., Williams, J. P., van der Marel, N., et al. 2016, ApJ, 828, 46

Anthonioz, F., Ménard, F., Pinte, C., et al. 2015, A\&A, 574, A41

Armitage, P. J. 2015, arXiv: 1509.06382

Bai, X.-N., \& Stone, J. M. 2010, ApJ, 722, 1437

Baraffe, I., Homeier, D., Allard, F., \& Chabrier, G. 2015, A\&A, 577, A42

Barenfeld, S. A., Carpenter, J. M., Ricci, L., \& Isella, A. 2016, ApJ, 827, 142

Beckwith, S. V. W., Henning, T., \& Nakagawa, Y. 2000, in Protostars and Planets IV, ed. V. Mannings, A. P. Boss, \& S. S. Russell (Tucson, AZ: Univ. of Arizona Press), 533

Bergin, E. A., Cleeves, L. I., Gorti, U., et al. 2013, Natur, 493, 644

Bergin, E. A., Du, F., Cleeves, L. I., et al. 2016, ApJ, 831, 101

Bohlin, R. C., Savage, B. D., \& Drake, J. F. 1978, ApJ, 224, 132

Boss, A. P. 1997, Sci, 276, 1836

Bruderer, S. 2013, A\&A, 559, A46

Bruderer, S., van der Marel, N., van Dishoeck, E. F., \& van Kempen, T. A. 2014, A\&A, 562, A26

Chapillon, E., Guilloteau, S., Dutrey, A., \& Piétu, V. 2008, A\&A, 488, 565 
Comerón, F. 2008, in Handbook of Star Forming Regions, Volume II: The Southern Sky ASP Monograph Publications, ed. B. Reipurth (Vol. 5; San Francisco, CA: ASP), 295

Cumming, A., Butler, R. P., Marcy, G. W., et al. 2008, PASP, 120, 531

Du, F., Bergin, E. A., Hogerheijde, M., et al. 2017, arXiv:1705.00799

Du, F., Bergin, E. A., \& Hogerheijde, M. R. 2015, ApJL, 807, L32

Dutrey, A., Guilloteau, S., \& Guelin, M. 1997, A\&A, 317, L55

Dutrey, A., Guilloteau, S., \& Simon, M. 2003, A\&A, 402, 1003

Espaillat, C., Muzerolle, J., Najita, J., et al. 2014, in Protostars and Planets VI, ed. H. Beuther, R. S. Klessen, C. P. Dullemond, \& T. Henning (Tucson, AZ: Univ. of Arizona Press), 497

Favre, C., Cleeves, L. I., Bergin, E. A., Qi, C., \& Blake, G. A. 2013, ApJL, 776, L38

Fedele, D., van den Ancker, M. E., Henning, T., Jayawardhana, R., \& Oliveira, J. M. 2010, A\&A, 510, A72

Feiden, G. A. 2016, A\&A, 593, A99

Feigelson, E. D., \& Babu, G. J. 2012, Modern Statistical Methods for Astronomy (Cambridge: Cambridge Univ. Press)

Foreman-Mackey, D., Hogg, D. W., Lang, D., \& Goodman, J. 2013, PASP, 125,306

France, K., Herczeg, G. J., McJunkin, M., \& Penton, S. V. 2014, ApJ, 794, 160

Gaia Collaboration, Brown, A. G. A., Vallenari, A., et al. 2016, arXiv:1609. 04172

Galli, P. A. B., Bertout, C., Teixeira, R., \& Ducourant, C. 2013, A\&A, 558, A77

Gorti, U., Hollenbach, D., Najita, J., \& Pascucci, I. 2011, ApJ, 735, 90

Haisch, K. E., Jr., Lada, E. A., \& Lada, C. J. 2001, ApJL, 553, L153

Harris, R. J., Andrews, S. M., Wilner, D. J., \& Kraus, A. L. 2012, ApJ, 751,115

Hartmann, L., Calvet, N., Gullbring, E., \& D’Alessio, P. 1998, ApJ, 495, 385

Hendler, N., Mulders, G. D., Pascucci, I., et al. 2017, arXiv:1705.01952

Henning, T., \& Semenov, D. 2013, ChRv, 113, 9016

Hernández, J., Hartmann, L., Calvet, N., et al. 2008, ApJ, 686, 1195

Howard, A. W., Marcy, G. W., Bryson, S. T., et al. 2012, ApJS, 201, 15

Howard, A. W., Marcy, G. W., Johnson, J. A., et al. 2010, Sci, 330, 653

Hughes, A. M., Wilner, D. J., Andrews, S. M., Qi, C., \& Hogerheijde, M. R. 2011, ApJ, 727, 85

Isella, A., Guidi, G., Testi, L., et al. 2016, PhRvL, 117, 251101

Isella, A., Testi, L., Natta, A., et al. 2007, A\&A, 469, 213

Johnson, J. A., Aller, K. M., Howard, A. W., \& Crepp, J. R. 2010, PASP, 122,905

Kama, M., Bruderer, S., Carney, M., et al. 2016a, A\&A, 588, A108

Kama, M., Bruderer, S., van Dishoeck, E. F., et al. 2016b, A\&A, 592, A83

Kamp, I., \& Dullemond, C. P. 2004, ApJ, 615, 991

Kelly, B. C. 2007, ApJ, 665, 1489

Kim, K. H., Watson, D. M., Manoj, P., et al. 2009, ApJ, 700, 1017

Kraus, A. L., Herczeg, G. J., Rizzuto, A. C., et al. 2017, ApJ, 838, 150

Kraus, A. L., Ireland, M. J., Hillenbrand, L. A., \& Martinache, F. 2012, ApJ, 745, 19

Krijt, S., Ciesla, F. J., \& Bergin, E. A. 2016, ApJ, 833, 285

Kusaka, T., Nakano, T., \& Hayashi, C. 1970, PThPh, 44, 1580

Lopez Martí, B., Jimenez Esteban, F., Bayo, A., et al. 2013, A\&A, 551, A46

Luhman, K. L. 2004, ApJ, 602, 816

Luhman, K. L., Allen, L. E., Allen, P. R., et al. 2008, ApJ, 675, 1375

Luhman, K. L., \& Mamajek, E. E. 2012, ApJ, 758, 31

Lyons, J. R., \& Young, E. D. 2005, Natur, 435, 317

Manara, C. F., Fedele, D., Herczeg, G. J., \& Teixeira, P. S. 2016a, A\&A, 585, A136

Manara, C. F., Rosotti, G., Testi, L., et al. 2016b, A\&A, 591, L3

Manara, C. F., Testi, L., Herczeg, G. J., et al. 2017, arXiv:1704.02842

Manoj, P., Kim, K. H., Furlan, E., et al. 2011, ApJS, 193, 11

Mayor, M., Marmier, M., Lovis, C., et al. 2011, arXiv:1109.2497
McClure, M., Bergin, T., Cleeves, I., et al. 2016, arXiv:1608.07817

Miotello, A., Bruderer, S., \& van Dishoeck, E. F. 2014, A\&A, 572, A96

Miotello, A., van Dishoeck, E. F., Kama, M., \& Bruderer, S. 2016, A\&A, 594, A85

Miotello, A., van Dishoeck, E. F., Williams, J. P., et al. 2017, A\&A, 599, A113

Mohanty, S., Greaves, J., Mortlock, D., et al. 2013, ApJ, 773, 168

Mordasini, C., Alibert, Y., Benz, W., Klahr, H., \& Henning, T. 2012, A\&A, 541, A97

Mulders, G. D., Pascucci, I., \& Apai, D. 2015a, ApJ, 798, 112

Mulders, G. D., Pascucci, I., \& Apai, D. 2015b, ApJ, 814, 130

Murphy, S. J., Lawson, W. A., \& Bessell, M. S. 2013, MNRAS, 435, 1325

Nguyen, D. C., Brandeker, A., van Kerkwijk, M. H., \& Jayawardhana, R. 2012, ApJ, 745, 119

Öberg, K. I., Murray-Clay, R., \& Bergin, E. A. 2011, ApJL, 743, L16

Obermeier, C., Koppenhoefer, J., Saglia, R. P., et al. 2016, A\&A, 587, A49

Pascucci, I., Testi, L., Herczeg, G. J., et al. 2016, ApJ, 831, 125

Pérez, L. M., Carpenter, J. M., Andrews, S. M., et al. 2016, Sci, 353, 1519

Perez, S., Casassus, S., Ménard, F., et al. 2015, ApJ, 798, 85

Pericaud, J., Di Folco, E., Dutrey, A., Guilloteau, S., \& Pietu, V. 2016, arXiv: 1612.06582

Petigura, E. A., Howard, A. W., \& Marcy, G. W. 2013, PNAS, 110, 19273

Pollack, J. B., Hubickyj, O., Bodenheimer, P., et al. 1996, Icar, 124, 62

Rodriguez, D. R., van der Plas, G., Kastner, J. H., et al. 2015, A\&A, 582, L5

Rosenfeld, K. A., Andrews, S. M., Wilner, D. J., Kastner, J. H., \& McClure, M. K. 2013, ApJ, 775, 136

Schegerer, A., Wolf, S., Voshchinnikov, N. V., Przygodda, F., \& Kessler-Silacci, J. E. 2006, A\&A, 456, 535

Schmidt, T. O. B., Vogt, N., Neuhäuser, R., Bedalov, A., \& Roell, T. 2013 , A\&A, 557, A80

Schwarz, K. R., Bergin, E. A., Cleeves, L. I., et al. 2016, ApJ, 823, 91

Smith, R. L., Pontoppidan, K. M., Young, E. D., Morris, M. R., \& van Dishoeck, E. F. 2009, ApJ, 701, 163

van der Marel, N., van Dishoeck, E. F., Bruderer, S., et al. 2013, Sci, 340, 1199

van der Marel, N., van Dishoeck, E. F., Bruderer, S., et al. 2016, A\&A, 585, A58

van der Marel, N., van Dishoeck, E. F., Bruderer, S., Pérez, L., \& Isella, A 2015, A\&A, 579, A106

van der Plas, G., Ménard, F., Ward-Duong, K., et al. 2016, ApJ, 819, 102

van Dishoeck, E. F., \& Black, J. H. 1988, ApJ, 334, 771

van Kempen, T. A., van Dishoeck, E. F., Güsten, R., et al. 2009, A\&A 507,1425

van Zadelhoff, G.-J., van Dishoeck, E. F., Thi, W.-F., \& Blake, G. A. 2001, A\&A, 377, 566

Voirin, J., Manara, C.F., \& Prusti, T. 2017, A\&A, submitted

Walsh, C., Nomura, H., Millar, T. J., \& Aikawa, Y. 2012, ApJ, 747, 114

Wang, H., \& Henning, T. 2006, ApJ, 643, 985

Weidenschilling, S. J. 1977, MNRAS, 180, 57

Wichmann, R., Krautter, J., Covino, E., et al. 1997, A\&A, 320, 185

Williams, J. P., \& Best, W. M. J. 2014, ApJ, 788, 59

Williams, J. P., \& Cieza, L. A. 2011, ARA\&A, 49, 67

Wilson, T. L., \& Rood, R. 1994, ARA\&A, 32, 191

Winn, J. N., \& Fabrycky, D. C. 2015, ARA\&A, 53, 409

Woitke, P., Kamp, I., \& Thi, W.-F. 2009, A\&A, 501, 383

Xu, R., Bai, X.-N., \& Öberg, K. 2017, ApJ, 835, 162

Youdin, A. N., \& Goodman, J. 2005, ApJ, 620, 459

Youdin, A. N., \& Shu, F. H. 2002, ApJ, 580, 494

Yu, M., Evans, N. J., Dodson-Robinson, S. E., Willacy, K., \& Turner, N. J. 2017, arXiv:1704.05508

Yu, M., Willacy, K., Dodson-Robinson, S. E., Turner, N. J., \& Evans, N. J., II 2016, ApJ, 822, 53

Zhang, K., Isella, A., Carpenter, J. M., \& Blake, G. A. 2014, ApJ, 791, 42 